Aus der Abteilung Klinische Neurophysiologie

(Prof. Dr. med. W. Paulus)

im Zentrum Neurologische Medizin

der Medizinischen Fakultät der Universität Göttingen

\title{
Einfluss verschiedener transkranieller Stimulationsverfahren auf die kortikale Exzitabilität
}

\author{
INAUGURAL - DISSERTATION \\ zur Erlangung des Doktorgrades \\ der Medizinischen Fakultät der \\ Georg-August-Universität zu Göttingen
}

vorgelegt von

Georg Fritzsche

aus

Jena

Göttingen 2010 
Dekan: Prof. Dr. med. C. Frömmel

I. Berichterstatterin: Prof. Dr. rer. nat. A. Antal

II. Berichterstatter/in: Prof. Dr. med. Brockmann

III. Berichterstatter/in:

Tag der mündlichen Prüfung: 17.11.2010 


\section{Verzeichnis der Abkürzungen}

$\begin{array}{ll}\text { A } & \text { Ampere } \\ \mathrm{Ag} & \text { Silber } \\ \mathrm{AgCl} & \text { Silberchlorid } \\ \mathrm{AMT} & \text { aktive motorische Reizschwelle } \\ \text { ANOVA } & \text { Analysis of Variance (Varianzanalyse) } \\ \mathrm{C} & \text { Coulomb } \\ \mathrm{cAMP} & \text { zyklisches Adenosinmonophosphat } \\ \mathrm{cm}^{2} & \text { Quadratzentimeter } \\ \mathrm{cTBS} & \text { kontinuierliche Thetaburststimulation } \\ \mathrm{EEG} & \text { Elektroenzephalografie } \\ \mathrm{EMG} & \text { Elektromyografie } \\ \mathrm{FDI} & \text { Musculus interosseus dorsalis I } \\ \mathrm{fMRT} & \text { funktionelle Magnetresonanztomografie } \\ \mathrm{Hz} & \text { Hertz } \\ \mathrm{iTBS} & \text { intermittierende Thetaburststimulation } \\ \text { imTBS } & \text { intermediäre Thetaburststimulation } \\ \mathrm{kHz} & \text { Kilohertz } \\ \text { LTD } & \text { Langzeithemmung } \\ \text { LTP } & \text { Langzeitpotentierung } \\ \mathrm{M} 1 & \text { primär-motorische Rinde } \\ \mathrm{mA} & \text { Milliampere } \\ \mathrm{MEP} & \text { Motorisch evozierte Potentiale } \\ \mu \mathrm{H} & \text { Mikrohenry } \\ \mathrm{min} & \text { Minuten } \\ \mathrm{mm} & \text { Millimeter } \\ \mathrm{MRT} & \text { Magnetresonanztomografie } \\ \mathrm{MSAP} & \text { Muskelsummenaktionspotential } \\ \mu \mathrm{V} & \text { Mikrovolt } \\ \mathrm{mV} & \text { Millivolt } \\ \mathrm{Na}{ }^{+} & \text {Natriumionen } \\ & \end{array}$




$\begin{array}{ll}\text { NMDA } & \text { N-Methyl-D-Aspartat } \\ \text { RMT } & \text { Motorschwelle in Ruhe } \\ \text { rTMS } & \text { repetitive transkranielle Magnetstimulation } \\ \text { S } & \text { Sekunde } \\ \text { sps } & \text { Abtastrate } \\ \text { tDCS } \wedge & \text { transkranielle Gleichstromstimulation } \\ \text { TES } & \text { transkranielle elektrische Stimulation } \\ \text { TMS } & \text { transkranielle Magnetstimulation } \\ \text { tRNS } & \text { transkranielle Rauschstromstimulation }\end{array}$




\section{Inhaltsverzeichnis}

INHALTSVERZEICHNIS

1. EINLEITUNG UND GRUNDLAGEN

1.1 INDUKTION UND MESSUNG VON NEUROPLASTIZITÄT MIT HILFE TRANSKRANIELLER STIMULATIONSVERFAHREN

1.1.1 TMS UND RTMS

1.1.2 TRANSKRANIELLE GLEICHSTROMSTIMULATION (TDCS)

1.1.3 INTERMITTIERENDE THETABURSTSTIMULATION (ITBS)

1.1.4 TRANSKRANIELLE RAUSCHSTROMSTIMULATION (TRNS)

1.2 SICHERHEITSASPEKTE

1.3 ZIELE

2. MATERIAL UND METHODEN

2.1 STUDIENTEILNEHMER

2.2 ELEKTROPHYSIOLOGISCHE MESSUNGEN

2.2.1 VORBEREITUNG

2.2.2 TMS-MESSUNGEN

2.2.3 SCHWELLENWERTE

2.3 STIMULATIONSTECHNIKEN

2.3.1 TDCS ANODAL $_{\text {An }}$

2.3.2 ITBS

2.3.3 TRNS

2.3.4 SHAM-STIMULATION

2.4 DATENAUSWERTUNG

2.4.1 STATISTISCHE AUSWERTUNG

3. ERGEBNISSE

3.1 DESKRIPTIVE STATISTIK

3.2 INTENSITÄT

3.3 VARIANZANALYSEN

3.3.1 TDCS ANODAL $_{\text {I }}$

3.3.2 ITBS

3.3.3 TRNS

\section{DISKUSSION}




\section{Einleitung und Grundlagen}

Eine der wichtigsten Eigenschaften des menschlichen Gehirns ist es, sich selbst strukturell und funktionell zu reorganisieren. Diese Funktion wird als Neuroplastizität beschrieben und ist unter anderem die Konsequenz aus Lern- oder Umwelteinflüssen, z.B. als dynamische Antwort auf ein Trauma oder als Resultat von Lernvorgängen (Elbert et al. 1995). Die Reorganisation erfolgt sowohl auf molekularer als auch auf zellulärer Ebene (Yuste und Bonhoeffer 2001). Entscheidend ist, dass dieses Potential nicht nur während der Entwicklungsperiode besteht, wie lange vermutet wurde, sondern auch im Erwachsenenalter (Rossini und Pauri 2000). Die Untersuchung von Neuroplastizität hängt, wie im Folgenden beschrieben, eng mit der Funktionsweise des Gehirns zusammen. Die Abhängigkeit neuronaler Signalübertragung von elektrischer Aktivität wurde erstmals mittels Untersuchungen am offenen Kortex von Affen und Hunden belegt (Ferrier 1875, Fritsch und Hitzig 1870). An diese ersten Befunde anschließend, fanden seit Anfang des vergangen Jahrhunderts systematische Untersuchungen mittels elektrischer Stimulation statt; erst an Primaten (Leyton und Sherrington 1917) und später intraoperativ an freigelegten Gehirnen neurochirurgischer Patienten (Foerster 1936, Penfield und Boldrey 1937, Penfield und Rasmussen 1950). Dies ermöglichte eine detaillierte funktionelle Kartierung des menschlichen Gehirns, welches bis dahin ausschließlich zytoarchitektonisch gegliedert werden konnte. Die weitere Untersuchung des menschlichen Gehirns mit non-invasiven Verfahren wurde ab 1980 möglich. Merton und Morton (1980) entwickelten das Verfahren der transkraniellen elektrischen Stimulation (TES) der menschlichen Großhirnrinde. Hierbei wurden kurze, hochintensive elektrische Stimuli verwendet, um die Nervenzellen des motorischen Kortex überschwellig zu reizen und damit Muskelsummenaktionspotentiale (MSAPs) auszulösen. Da dieses Verfahren bedingt durch die hohen elektrischen Spannungen gleichzeitig die Nozizeptoren (Schmerzrezeptoren) der Kopfhaut reizt, war der routinemäßige Einsatz dieser Technik begrenzt. Kurze Zeit später wurde durch Barker et al. (1985 a, b, 1987) die transkranielle Magnetstimulation (TMS) eingeführt. Wie im Folgenden beschrieben, ist die TMS in der Lage, ähnlich effektiv und gleichzeitig schmerzfrei die Hirnrinde zu aktivieren. Beide Verfahren erzielen eine kurzfristige 
überschwellige Aktivierung (im Bereich von Millisekunden) zerebraler Neuronenverbände. Jedoch sind für die Betrachtung der Neuroplastizität vor allem längerfristige und beständige Erregbarkeitsänderungen (im Minuten- bis Stundenbereich) von Interesse. Das bekannteste Tierexperiment bezüglich lang anhaltender neuroplastischer Aktivitätsveränderungen entwickelten Bliss und Lomo (1973). Sie erzeugten diese Modulationen durch präsynaptische elektrische Stimulation an hippokampalen Hirnschnitten. Nach hochfrequenter elektrischer Stimulation entwickelte sich hierbei eine lang anhaltende Erregbarkeits- bzw. Aktivitätserhöhung, nach niederfrequenter Stimulation die entgegengesetzte Veränderung. Diese Vorgänge wurden als long-term potentiation (LTP) und long-term depression (LTD), respektive, beschrieben. Weitere Untersuchungen, sowohl in vivo als auch in vitro, erzeugten ähnliche Phänomene (Buonomano und Merzenich 1998, Castro-Almancos und Calcagnotto 1999, Eysel et al. 1998, Hess und Donoghue 1994, Krug et al. 1984).

Auch im Humanexperiment ist es mittlerweile möglich, LTP- und LTD-ähnliche Effekte mit verschiedenen Techniken zur Hirnstimulation auszulösen (vor allem mit transkranieller Stromstimulation und repetitiver transkranieller Magnetstimulation). Die vorliegende Studie beschäftigt sich mit dem Vergleich der Nacheffekte zweier seit längerem bekannten Stimulationsverfahren, im Vergleich zu einem kürzlich entwickelten Verfahren der transkraniellen Stimulation der Hirnrinde.

\subsection{Induktion und Messung von Neuroplastizität mit Hilfe transkranieller Stimulationsverfahren}

\subsubsection{TMS und rTMS}

Die für diese Studie benutzte Technik der transkraniellen Magnetstimulation (TMS) beruht auf dem Faraday'schen Gesetz der elektromagnetischen Induktion. Dabei wird in einer zirkulären (hier: achtförmigen) Kupferspule durch starke Stromstöße (bis 10000 A) ein transientes Magnetfeld erzeugt, welches von einigen hundert Mikrosekunden Dauer ist. Dieses passagere Magnetfeld durchdringt die intakte Schädelkalotte und sämtliche Hirnhäute. Es erreicht das darunterliegende Hirngewebe ohne nennenswerte Abschwächung. Da die Neuronen einen stationären Leiter 
darstellen, wird in innen ein Stromfluss induziert, welcher bei überschwelliger Reizung ein Aktionspotential durch Ladungsverschiebungen über den Zellmembranen auslösen kann. Maßgeblich verantwortlich für die Größe des Stromflusses in den Nervenzellen ist ein möglichst kurzer Magnetimpuls, da sich die induzierte Spannung proportional zur Änderungsgeschwindigkeit des Magnetfeldes verhält. Das Magnetfeld muss sich also schnell aufbauen, jedoch sollte es möglichst langsam wieder abgebaut werden, da sonst ein zweiter Stromfluss in die entgegen gesetzte Richtung induziert wird (Schubert 1997, Rossini et al. 1991). Mit hohen Stimulationsintensitäten führt die Erregung von Pyramidenbahnzellen direkt zu einem Aktionspotential, bei mittleren Intensitäten werden die Pyramidenbahnneurone hauptsächlich indirekt über Interneurone erregt (Edgley et al. 1997). Mit dieser Technik ist es möglich, die Hirnrinde unter anderem am Repräsentationsareal eines Muskels fokal zu erregen und im Weiteren die Erregung mittels EMG-Ableitungen an diesem Muskel zu quantifizieren (motorisch evozierte Potentiale, MEP). Aus diesem Grund eignet sich die TMS besonders für die Betrachtung von kurzzeitigen und länger anhaltenden Aktivitätsveränderungen im motorischen Kortex.

Die repetitive transkranielle Magnetstimulation (rTMS) ist ein Verfahren zur Neuromodulation, welches Anfang der 90er Jahre entwickelt wurde (Pascual-Leone et al. 1994). Dabei werden in regelmäßigen Abständen in einem definierten Zeitraum eine bestimmte Anzahl von gruppierten oder ungruppierten Magnetimpulsen abgegeben. Dies kann zu einer Veränderung der kortikalen Erregbarkeit führen (Siebner und Rothwell 2003). Den Haupteinfluss auf die Richtung der Exzitabilitätsveränderung bei der konventionellen rTMS hat die Frequenz der verabreichten Stimuli. Hohe Frequenzen (> $5 \mathrm{~Hz}$ ) führen zu einer Bahnung (Peinemann et al. 2004, Quartarone et al. 2005), Frequenzen im Bereich von 0,3 - $1 \mathrm{~Hz}$ hemmen die kortikale Erregbarkeit (Chen et al. 1997, Touge et al. 2001, Wassermann et al. 1996b). Je nach Höhe der Intensität halten diese Effekte für mehrere Minuten nach Ende der rTMS an (Chen et al. 1997, Fitzgerald et al. 2002). 


\subsubsection{Transkranielle Gleichstromstimulation (tDCS)}

Die transkranielle Gleichstromstimulation ist eine nicht-invasive Methode zur Modulation der Exzitabilität des menschlichen Kortex (Nitsche und Paulus 2000). Systematische Tierversuche an anästhesierten Ratten zeigten schon vor Jahrzehnten den Einfluss von schwachem Gleichstrom, hier appliziert mit intrazerebralen oder epiduralen Elektroden, auf die Erregbarkeit des Kortex (Bindman et al. 1964). Mit diesem Verfahren führte die anodale Stimulation zu einer gesteigerten Erregbarkeit, die kathodale Anwendung zu einer Verminderung (Bindman et al. 1962, Purpura und McMurtry 1965). Diese Erkenntnisse führten zu Untersuchungen, bei denen psychiatrisch Erkrankte mittels transkraniell applizierten Gleichstroms behandelt wurden (Costain et al. 1964, Carney 1969). Die in diesen Studien erzielten positiven Ergebnisse konnten jedoch in weiteren Untersuchungen nicht reproduziert werden, für eine Übersicht dazu siehe Lolas (1977). Die Bezeichnung der Stimulation (anodal oder kathodal) bezieht sich hier und im folgenden Text immer auf den Ort der Stimulationselektrode. Dabei ist die Positionierung der Elektroden von großer Wichtigkeit. Zur effektiven Stimulation des motorischen Kortex hat sich nach systematischer Untersuchung nur folgende Anordnung bewährt (Nitsche und Paulus 2000): die Stimulationselektrode über dem zu stimulierenden motorischen Areal, die indifferente Elektrode über der kontralateralen Orbita. Inzwischen sind die Effekte der tDCS in einer Reihe von Studien an Probanden überprüft wurden (Nitsche et al. 2008). So konnten erstens für die mindestens drei Minuten angebrachte anodale Stimulation längerfristige Erregungssteigerungen im stimulierten Kortexareal nach Stimulationsende mittels TMS nachgewiesen werden und zweitens eine signifikante Verminderung der Erregbarkeit bei kathodaler Stimulation (Nitsche und Paulus 2000). Später wurde von Nitsche et al. (2007) gezeigt, dass neben der optimalen Position der Elektroden die Stromdichte (Stromstärke $[\mathrm{A}]$ / Elektrodenfläche [cm2]) entscheidend für die Effektivität der Stimulation ist. So verkleinerten sie die Stimulationselektroden auf ein Zehntel der Größe der üblicherweise verwendeten Elektroden bei gleichbleibender Stromdichte. Dadurch erzielten sie fokalere Effekte auf den motorischen Kortex und mussten gleichzeitig keine quantitativen Verluste bei der Stimulation hinnehmen.

Die tDCS kommt inzwischen auch häufig in klinischen Versuchen zur Anwendung. So ist es Fregni et al. (2005) sowie Hummel und Cohen (2005) gelungen, 
die motorischen Funktionen von Schlaganfallpatienten zu verbessern. Sie nutzten dazu die kathodale Gleichstromstimulation über der betroffenen Hemisphäre und anodale tDCS über der Gegenseite. Weitere Studien beschäftigen sich mit der Behandlung von Depressionen mit Hilfe der anodalen tDCS. Dabei gibt es erfolgversprechende Resultate, so haben Boggio et al. (2008) bei 40 Patienten mit Depression eine signifikante Verbesserung der Beschwerden festgestellt, gemessen mit verschiedenen Depressions-Scores. Neueste Doppelblindstudien gegen Placebo-Stimulation bestätigen die Wirksamkeit der andolen tDCS im Bezug auf die Lebensqualität und die Schmerzwahrnehmung bei Patienten mit Multipler Sklerose (Mori et al. 2009). Damit ist die tDCS ein erfolgreiches Werkzeug bei der Erforschung der Neuroplastizität und mindestens eine gute Unterstützung bei der Behandlung verschiedener neurologischer Erkrankungen.

\subsubsection{Intermittierende Thetaburststimulation (iTBS)}

Die intermittent theta burst stimulation ist ein relativ neues Stimulationsprotokoll aus der Gruppe der repetitiven transkraniellen Magnetstimulation (rTMS). Huang et al. (2005) entwickelten das neue iTBS-Protokoll mit Verwendung von salvenartigen repetitiven Stimuli, welches sich an ein tierexperimentelles Protokoll zur Erzeugung von LTP und LTD anlehnte (Larson und Lynch 1986). Das Protokoll von Huang et al. (2005) besteht aus hochfrequenten Dreiersalven (engl. bursts) mit einer Frequenz von $50 \mathrm{~Hz}$, die sich alle $200 \mathrm{~ms}$ (5 Hz, sog. Theta-Bereich) wiederholen. Das Besondere hierbei ist, dass die Stimuli nicht kontinuierlich verabreicht werden, sondern intermittierend. Nach einem Stimulationsblock von 2 Sekunden folgt eine Pause von 8 Sekunden. Entscheidend für die Auswirkung auf die Exzitabilität des stimulierten Kortexareals ist der Abstand der Stimulationsblöcke. Bei der kontinuierlichen Thetaburststimulation (cTBS) folgt ein Stimulationsblock dem nächsten ohne längere Pausen. Daraus resultieren LTD-ähnliche Effekte. Überwacht wurden die Aktivitäten über die Amplitude der motorisch evozierten Potentiale (MEPs). Thetaburststimulation ohne Unterbrechungen (cTBS) führt zu einer signifikanten Reduzierung der MEP-Amplituden aufgrund der spezifischen Hemmung eines intrazerebralen Reizkreislaufs (Di Lazzaro et al 2005). Fügt man aber die oben genannten 8 Sekunden Pause ein (und erhält iTBS), 
kommt es zu bahnenden Effekten auf die MEP-Amplituden bis ungefähr 15 min nach der Stimulation (Huang et al. 2005). Außerdem konnte gezeigt werden, dass neben M1 auch in dem menschlichen prämotorischen (Mochizuki et al 2005) und dem visuellen Kortex (Franca et al 2006) durch Thetaburststimulation ein Einfluss auf die Erregbarkeit stattfindet. Interessanterweise zeigt intermediäre Thetaburststimulation (imTBS) überhaupt keine Effekte. Im Vergleich zur konventionellen rTMS mit gleichmäßiger Stimuliabfolge erfordert die iTBS eine niedrigere Stimulationsintensität von nur $80 \%$ der aktiven motorischen Reizschwelle (AMT) und kürzere Stimulationsdauern von nur 20$190 \mathrm{~s}$.

iTBS

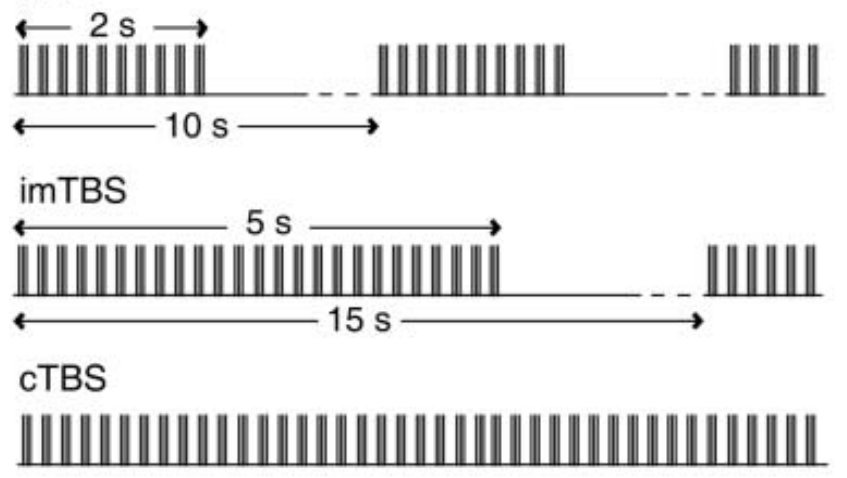

\section{Abbildung 1: Thetaburststimulation}

Schematische Darstellung der Thetaburststimulation (TBS) nach Huang et al (2005) mit intermittierender TBS, intermediärer TBS und kontinuierlicher TBS.

Inzwischen gibt es auch eine Reihe von Studien zu therapeutischen Interventionen mit dieser Technik bei Neurologischen Erkrankungen. Talelli et al.(2007) untersuchten Schlaganfallpatienten mit unvollständiger Ausheilung von Paresen der oberen Extremität. Eine einzelne Sitzung iTBS (600 Pulse) über der betroffenen Hemisphäre verbesserte die motorische Funktion der paretischen Hand in Bezug auf die Reaktionszeit für $30 \mathrm{~min}$ verglichen mit einer Sham-Stimulation und einer cTBS. Demgegenüber steht der Versuch, in einer einzelnen Sitzung iTBS Tinnitus-Symptome zu lindern (Poreisz et al. 2009). Diese Anwendung führte nicht zu einer Verbesserung. Positive Ergebnisse zur Verbesserung von spastischen Paresen der unteren Extremität bei Patienten mit Multipler Sklerose (MS) erbrachte die tägliche Anwendung der iTBS über 2 Wochen (Mori et al. 2009). 
In einem Konsensus (Huang et al. 2009) zu verschiedenen neuen Stimulationsmethoden, unter Anderem der iTBS, wurde darauf hingewiesen, dass weitere Untersuchungen zum physiologischen Wirkmechanismus und der Effektivität in den unterschiedlichen klinischen Anwendungsgebieten nötig sind.

\subsubsection{Transkranielle Rauschstromstimulation (tRNS)}

Die transkranielle Rauschstromstimulation (transcranial random noise stimulation) ist eine neue Technik zur Steigerung kortikaler Exzitabilität, welche von Terney et al. (2008) vorgestellt wurde. Im Gegensatz zur tDCS wird hier kein Gleichstrom angewendet, sondern das entsprechende Hirnareal mit einem hochfrequenten zufälligen Strompegel stimuliert. Damit ließen sich ebenfalls neuroplastische Veränderungen erzeugen, ähnlich denen, die aus den tDCS Protokollen bekannt sind. Darüber hinaus hat die Rauschstromstimulation weitere Vorteile, da hier nicht die Richtung des Stromflusses für den Effekt entscheidend ist. Bei der Stimulation der gefurchten Kortexoberfläche mit Strom, der nur in eine Richtung fließt (z.B. anodale tDCS), wird auf einer Seite eines Gyrus ein exzitatorischer Nacheffekt induziert. Auf der gegenüberliegenden Seite wird unausweichlich ein inhibitorischer Effekt ausgelöst. Benutzt man die tRNS, sind nur exzitatorische Langzeiteffekte zu beobachten (Terney et al. 2008). Des Weiteren können nun auch Nervenzellpopulationen stimuliert werden, deren symmetrische Dendritenbäume nur tangential vom angelegten elektrischen Feld berührt werden, ohne dass es zu neutralisierenden Strömen in die entgegengesetzte Richtung kommt.

Eine weitere Studie fand eine vorübergehende Abnahme der BOLD-Aktivität im funktionellen MRT (fMRT) nach vierminütiger tRNS (Chaieb et al. 2009) im stimulierten Kortexareal. Das ist insofern interessant, als dass die TMS-Untersuchungen von Terney et al. (2008) bei zehnminütiger tRNS ausschließlich die Zunahme von MEP-Amplituden, und damit eine Aktivitätssteigerung beobachten konnten.

Die vorliegende Studie soll einen Beitrag zur Evaluation der Effizienz dieser neuen Methode leisten. 


\subsection{Sicherheitsaspekte}

Seit ca. 20 Jahren hat die TMS einen festen Platz in der klinischen Routinediagnostik. Bis zum heutigen Zeitpunkt sind keine gravierenden oder bedrohlichen Nebenwirkungen wie zum Beispiel strukturelle Hirnschädigungen bekannt geworden. Dennoch birgt die Anwendung der TMS potentielle Gefahren, insbesondere bei falscher Anwendung mit sehr hohen Intensitäten und langer Anwendungsdauer. Einzelne Fälle vom Auftreten epileptischer Anfälle bei der Benutzung hoher Intensitäten und repetitiver TMS sind in einzelnen Studien beschrieben worden (Pascual-Leone et al. 1993, Nowak et al. 2006). Um diese Risiken zu minimieren, wurden in der internationalen wissenschaftlichen Gemeinschaft Richtlinien für die Anwendung der TMS und der rTMS aufgestellt. Grundlegende Anwendungsregeln nannte der "Workshop on the safety of repetitive transcranial magnetic stimulation" im Jahre 1996 und 2008 unter Berücksichtigung der Erfahrungen bezüglich Nebenwirkungen der TMS (Anand und Hotson 2002, Brandt et al. 1997, Pascual-Leone et al. 1993, Wassermann 1998, Wassermann et al. 1996a, Barker 1991). Demnach gilt es, die Probanden gründlich aufzuklären, schriftlich einwilligen zu lassen und die Richtlinien zur Dauer und Intensität vor allem bei repetitiven Magnetstimulationen $\mathrm{zu}$ beachten. Alle in den genannten Studien postulierten absoluten und relativen Kontraindikationen betrachteten wir als absolute Ausschlusskriterien für die Teilnahme an dieser Studie. Die empfohlenen Grenzwerte für Stimulationsfrequenz, -dauer und -intensität wurden in der vorliegenden Studie zu keiner Zeit überschritten. Die Stimulationsintensitäten richteten sich nach den individuellen kortikalen Erregungsschwellen. Diese wurden mit Hilfe der sogenannten individuellen Motorschwelle in Ruhe (resting motor treshold, RMT) oder im aktivierten Zustand (active motor treshold, AMT) bestimmt. Die RMT ist als geringste Intensität definiert, auf die das individuelle Gehirn mit einer messbaren motorischen Reaktion in Form eines MEP reagiert. Sie entspricht nach Pascual-Leone et al. (1998) der Membran-Erregbarkeit der horizontalen pyramidalen Neuronen.

Zur Evaluation der Sicherheit wurde der Einfluss von iTBS und cTBS auf die Kognition, Stimmung und das Ruhe-EEG von Grossheinrich et al. (2009) an 24 gesunden Probanden untersucht. Stimuliert wurden der dorsolaterale präfrontale Kortex (DFPLC) und der mediale präfrontale Kortex (mPFC). Sie stellten keine gravierenden Nebenwirkungen und keinen Einfluss auf die kognitiven Fähigkeiten fest. Lediglich das 
Auftreten vagaler Symptome, die spontan nach kurzer Ruhephase sistierten, wurde von 3 Probanden berichtet. Das Ruhe-EEG zeigte in der genannten Studie weder Krampfnoch epileptiforme Aktivität nach der Stimulation.

Für die tDCS gibt es bislang keine Studien, die systematisch die Grenzen einer sicheren Stimulation definiert haben. Jedoch wurden Kriterien für eine sichere Stimulation aus Literaturrecherchen abgeleitet (Nitsche et al. 2003). Ein möglicher Schädigungsmechanismus, wenn auch irrelevant für Schädigungen des Gehirns, ist die Entstehung toxischer Reaktionsprodukte an der Kontaktstelle zwischen Elektroden und Kopfhaut. Dies wird in der vorliegenden Studie aber durch Verwendung metallfreier Schwammelektroden verhindert. Außerdem kann es prinzipiell bei langer Stimulationsdauer mit hohen Intensitäten zu Hitzentwicklung und konsekutiver Gewebsschädigung an der Kontaktstelle kommen. In einer von Nitsche und Paulus (2000) vorgestellten Studie wurde dies jedoch für das angewendete Stimulationsprotokoll ausgeschlossen. Entscheidend für die Effektivität der tDCS und somit auch für potentielle Schädigungen des Gehirngewebes ist die Stromdichte (Stromstärke [A] / Elektrodenfläche [cm2]) und die Dauer der Stimulation. McCreery et al. (1990) zeigten im Tierversuch, dass Stromdichten von unter $25 \mathrm{~mA} / \mathrm{cm} 2$ nicht zu Gewebeschädigungen führen. Die zusätzliche Betrachtung der Stimulationsdauer wird in der Gesamtladung berücksichtigt. Die Schädigungsgrenze liegt hier bei $216 \mathrm{C} / \mathrm{cm} 2$ (Yuen et al. 1981). In dem in dieser Studie angewendeten Stimulationsprotokoll lagen die Stromdichte mit $0,0625 \mathrm{~mA} / \mathrm{cm} 2$ und die Gesamtladung mit $0,0375 \mathrm{C} / \mathrm{cm} 2$ deutlich darunter.

Für die Sicherheit der Methode spricht auch eine große Studie, welche die Nebenwirkungen der tDCS mittels eines Fragebogens an über 100 Probanden untersuchte. Ernste Nebenwirkungen wurden nicht beobachtet, nur 11\% der Probanden verspürten leichte Kopfschmerzen nach der Stimulation, außerdem wurde über Kribbeln und Jucken während der Stimulation berichtet. Über Übelkeit berichteten nur 2,9\% der Befragten, insgesamt wurde die Prozedur nur von wenigen als leicht unangenehm bewertet und die Mehrheit der Probanden bemerkte keinen Unterschied zwischen anodaler, kathodaler oder Plazebostimulation (Poreisz et al. 2007).

Zur Beurteilung der Sicherheit der transkraniellen Rauschstromstimulation wurde von Terney et al. (2008) die neuronenspezifische Enolase (NSE) im Serum gemessen. 
Diese ist ein sensitiver Marker für die Schädigung von Neuronen (Steinhoff et al. 1999) bei verschiedenen neurologischen Erkrankungen. Die NSE war auch bei zehnminütiger Rauschstromstimulation an acht aufeinander folgenden Tagen nicht erhöht. Dies lässt die begründete Vermutung zu, dass eine limitierte Anwendung der tRNS als sicher gilt.

\subsection{Ziele}

Die Effekte der tDCS und der iTBS auf die Neuroplastizität sind in einer Reihe von Studien untersucht worden. Die anodale Konfiguration der Stimulationselektroden bei der tDCS und intermittierende Magnetstimulationsprotokolle der iTBS haben exzitatorische Effekte auf den menschlichen Kortex (Nitsche et al. 2008, Huang et al. 2005). Die Effektivität der 2008 neu eingeführten tRNS gegenüber einer ShamStimulation konnte von Terney et al. (2008) gezeigt werden. Das Ziel der vorliegenden Studie war es, die Effekte der einzelnen Stimulationsarten untereinander zu vergleichen, indem sie an derselben Gruppe von Studienteilnehmern angewendet wurden. Dies sollte hinsichtlich des Betrages der Erregbarkeitserhöhung und der Dauer der länger anhaltenden Exzitabilitätsveränderungen untersucht werden. Die Untersuchung sollte versuchen, weitere Hinweise auf die physiologischen Mechanismen der Exzitabilitätserhöhung zu finden die im Bereich der Veränderung des Ruhemembranpotentials und der synaptischen Effizienz der stimulierten Neuronen vermutet werden. Des Weiteren sollte diese Studie helfen, mögliche klinische Anwendungen der einzelnen Stimulationsarten zu evaluieren. 


\section{Material und Methoden}

\subsection{Studienteilnehmer}

An der vorliegenden Studie, die an der Universitätsmedizin Göttingen, Abteilung Klinische Neurophysiologie durchgeführt wurde, nahmen zwölf Probanden (sechs weiblich) im Alter zwischen 23 und 38 Jahren (Durchschnitt \pm Standardabweichung (SD): 25,67 $\pm 4,14$ Jahren) teil. Alle Probanden waren Rechtshänder (ermittelt durch das Edinburgh Inventory; Oldfield 1971) und wurden durch Aushänge im Bereich der Universitätsbibliothek, durch persönliche Ansprache oder aus Probandendatenbanken rekrutiert. Die Studie wurde von der ortsansässigen Ethikkommission genehmigt $(14 / 1 / 00 ; 8 / 7 / 06 ; 21 / 3 / 04)$ und erfolgte unter den ethischen Standards der Deklaration von Helsinki (1964).

Ausschlusskriterien zur Teilnahme anhand der allgemeinen Sicherheitsrichtlinien für TMS (Anand und Hotson. 2002, Pascual-Leone et al. 1993, Wassermann 1998, Wassermann et al. 1996b, Barker 1991, Brandt et al. 1997) waren: Epilepsie, Migräne, intrakranielle Metallimplantate jeder Art, das Tragen eines Herzschrittmachers, regelmäßige Einnahme jeglicher Medikamente (außer Antibabypille), Schwangerschaft, sonstige neurologische und psychiatrische Erkrankungen sowie schwere und/oder chronische körperliche Erkrankungen. Alle Probanden erhielten für ihre Teilnahme eine Aufwandsentschädigung. Vor Beginn der experimentellen Untersuchung wurden die Probanden mehrfach schriftlich und mündlich über den Versuchsablauf, mögliche Risiken und über die Möglichkeit, den Versuch jederzeit beenden zu können, aufgeklärt (Siehe Anhang 1 und 2).

\subsection{Elektrophysiologische Messungen}

\subsubsection{Vorbereitung}

Die Probanden wurden während der Messungen in bequemer, halbsitzender Position in einem umgebauten Zahnarztsessel mit verstellbarer Lehne und verstellbarer Kopfstütze positioniert. An der rechten Hand wurden zwei $\mathrm{Ag} / \mathrm{AgCl}-$ 
Oberflächenelektroden (Durchmesser 9mm) zur Ableitung der Muskelsummenaktionspotentiale (MSAP) mittels Elektromyogramm (EMG) angebracht; die differente Elektrode auf dem Muskelbauch des Musculus interosseus dorsalis I (FDI) und die indifferente über dem entsprechenden Sehnenansatz an der Grundphalanx des II. Strahls. Eine Erdungselektrode wurde am Unterarm positioniert. Das Rohsignal wurde verstärkt und mit einem Tiefenpassfilter $(2 \mathrm{kHz})$ gefiltert. Das Signal wurde im Anschluss über einen micro 1401 analog-zu-digital Wandler (Cambridge Electronic Design, Cambridge, UK) mit einer Wechselrate von $5 \mathrm{kHz}$ digitalisiert und unter Kontrolle der Software Signal (Cambridge Electronic, Version 2.13) auf einem IBM®-kompatiblen PC zur weiteren Analyse gespeichert. Um die absolute muskuläre Entspannung der rechten oberen Extremität zu gewährleisten, wurde den Probanden, immer wenn nötig, ein akustisches Feedback über die EMG-Aktivität gegeben.

\subsubsection{TMS-Messungen}

Die im Verlauf der experimentellen Untersuchung verwendeten monophasischen Magnetimpulse wurden mittels eines Magstim 200 Magnetstimulators generiert (Magstim Company, Whiteland, Wales, UK). Kombiniert wurde der Stimulator mit einer achtförmigen Magstim-Double-70-mm-Spule (Durchmesser der Spulenwindungen durchschnittlich $70 \mathrm{~mm}$ ). Diese Kombination erreicht eine maximale Feldstärke von 2,2 Tesla mit einer durchschnittlichen Selbstinduktion von $16,35 \mu \mathrm{H}$.

Während der experimentellen Untersuchungen wurde die oben genannte TMSSpule tangential zum Kopf gehalten in einem Winkel von ca. 45 Grad zur sagittalen Achse, den Griff nach posterior-lateral zeigend (Abbildung 2). Um die optimale Position zu finden, wurde die Spule unter der Abgabe von Einzel-Puls-Testreizen in kleinsten Schritten über der M1-Region der linken Hemisphäre bewegt. Bei Misserfolg, d.h. keine oder unzureichende EMG-Aktivität, wurde langsam die Intensität der Testreize erhöht und erneut die optimale Spulenposition gesucht. Diese wurde am Ort der größten und stabilsten MEP-Amplituden gefunden und entsprach dem Repräsentationsareal des FDI. Die entsprechende Stelle wurde zur genauen Repositionierung farblich markiert. Die Spule wurde während jeder Messung an dieser Position mit beiden Händen des Untersuchers fixiert. 


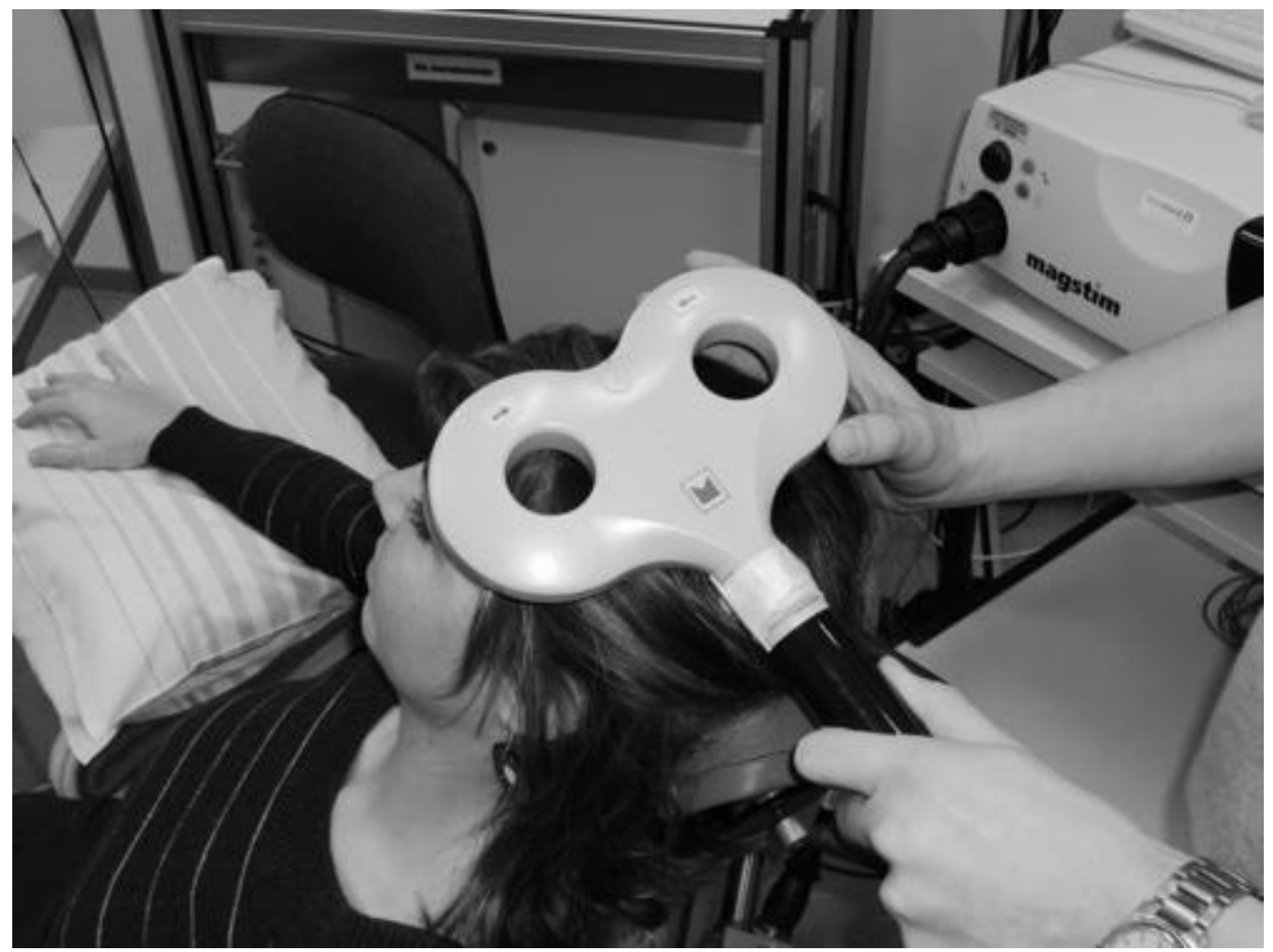

Abbildung 2: Spulenposition während der TMS-Messungen mit dem Griff nach posterolateral in einem Winkel von etwa 45 Grad zur Sagittalachse.

\subsubsection{Schwellenwerte}

Die Probanden wurden im Abstand von mindestens vier Tagen einbestellt. Nachdem die Vorbereitungen gewissenhaft abgeschlossen wurden, erfolgte die Bestimmung der Schwellenwerte nach den Sicherheitsrichtlinien von 1996 (Anand und Hotson 2002, Brandt et al. 1997, Pascual-Leone et al. 1993, Wassermann 1998, Wassermann et al. 1996b, Barker 1991). Dies ist nötig, da jedes Gehirn individuell auf elektromagnetische Stimulation reagiert.

Begonnen wurde immer mit der motorischen Ruheschwelle (resting motor threshold, RMT). Zur Ermittlung der RMT wurde ein Testreiz appliziert, der sicher oberhalb derselben lag. In 1\%igen Schritten wurde die Leistung des Stimulators solange reduziert, bis nur noch vier von sechs Impulsen eine MEP-Amplitude von $50 \mu \mathrm{V}$ hervorriefen. Die in Prozent angegebene Leistung des Stimulators stellte die RMT dar. 


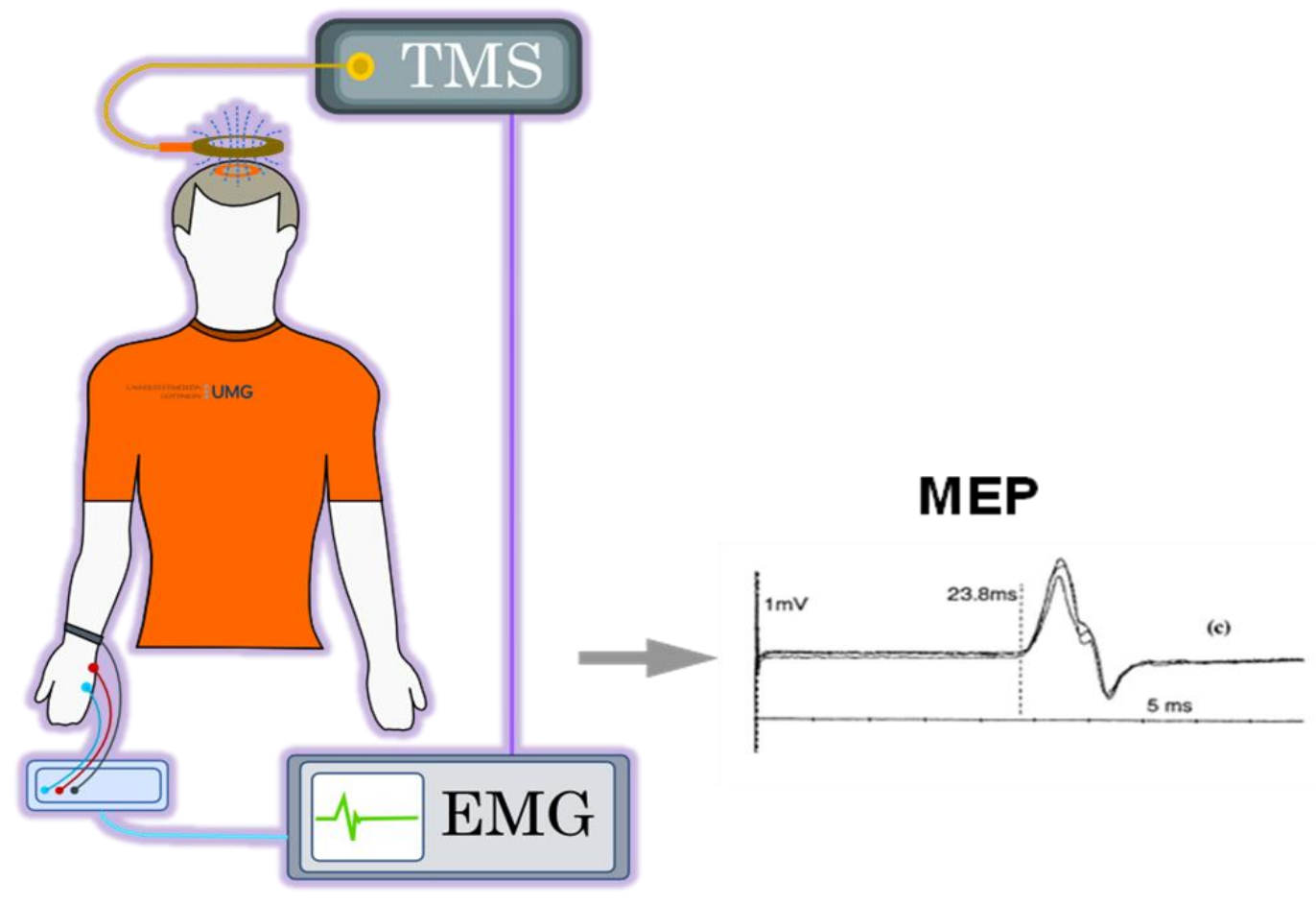

\section{Abbildung 3: Versuchsaufbau}

Der Magnetpuls aus der TMS-Spule erzeugt ein Muskelsummenaktionspotential, welches mithilfe der EMG-Ableitung als motorisch evoziertes Potential (MEP) graphisch dargestellt werden kann.

Es folgte die Ermittlung der aktiven motorischen Schwelle (active motor threshold, AMT). Der Proband wurde gebeten, eine isometrische Kontraktion des FDI unter Zuhilfenahme einer Pflasterrolle zwischen Daumen und Zeigefinger aufzubauen. Unter der Applikation von Testreizen wurde wiederum die Leistung des Stimulators reduziert, bis nur noch vier von sechs Impulsen eine verlässliche MEP-Antwort von ca. $200 \mu \mathrm{V}$ auslösten. Für die geplante iTBS Stimulation wurde dieser Vorgang mit dem Super Rapid Stimulator wiederholt, da später mit $80 \%$ der AMT stimuliert werden sollte.

Nach der Bestimmung der Schwellenwerte folgte das Aufzeichnen der Baseline. Dazu wurde die Intensität der Einzelpulse so gewählt, dass die erzeugten MEPAmplituden einen Peak-to-Peak-Wert von durchschnittlich $1 \mathrm{mV}$ ergaben. Dies wurde nach der Aufzeichnung umgehend überprüft, indem die im Programm "Signal“ dargestellten MEPs mit der Software „NuCursor" analysiert wurden. Der so errechnete Mittelwert aus den erzeugten MEP-Amplituden sollte möglichst genau bei $1 \mathrm{mV}$ liegen. 
Die Baseline, sowie jeder folgende Messblock, bestanden aus 40 Einzelpulsstimuli mit einer Frequenz von $0,25 \mathrm{~Hz}$.

\subsection{Stimulationstechniken}

Nach der Baseline-Bestimmung erfolgte die tDCS-Stimulation, die tRNSStimulation, die iTBS-Stimulation oder die Sham-Stimulation. Die Art der angewandten Stimulation war, bis auf die iTBS, für den Probanden nicht ersichtlich. Als Stimulationselektroden für die tDCS, tRNS und Sham-Stimulation wurden platte Gummielektroden verwendet. Diese befanden sich in einem mit 0,9\%iger Kochsalzlösung getränkten Schwamm. Die Stimulationselektrode $(4 \times 4 \mathrm{~cm})$ wurde über dem zuvor ermittelten Repräsentationsareal des FDI mit Gummibändern fixiert, ebenso die Referenzelektrode $(6 \times 14 \mathrm{~cm}$ ) frontopolar über der kontralateralen Orbita (Nitsche und Paulus 2000).

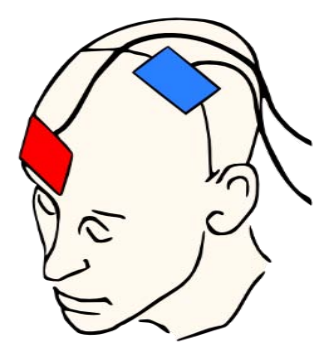

\section{Abbilduna 4: Anordnuna der Stimulationselektroden}

Der Erfolg der Stimulation wurde über eine im Stimulationsgerät integrierte Widerstandsmessung durchgehend überprüft. Nach Beendigung der Stimulation erfolgte unmittelbar die erste Nachmessung durch 40 TMS-Stimuli mit $0,25 \mathrm{~Hz}$. Die Intensität richtete sich nach der Baseline. Weitere Messungen zur Untersuchung der Nacheffekte wurden nach gleichem Schema fünf, zehn, 20, 30, 40, 50, 60 und 90 Minuten nach Stimulationsende durchgeführt (Abbildung Versuchsaufbau). 


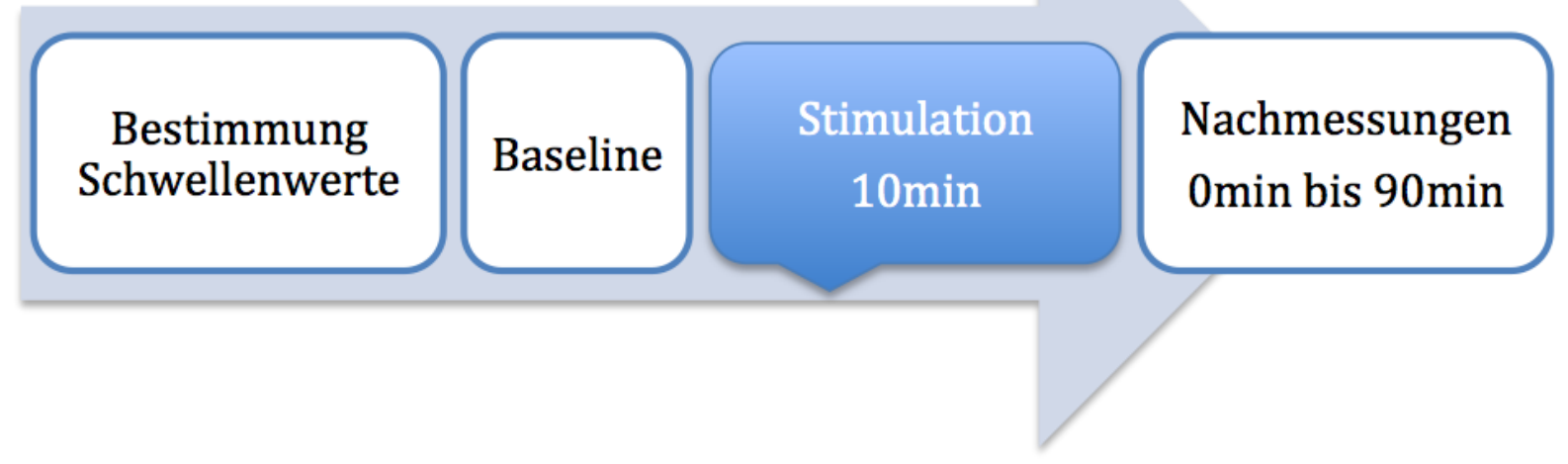

\section{Abbildung 5: Versuchsablauf}

Standardisierter Ablauf aller Versuche: Schwellenwertbestimmung, Aufzeichnung der Baseline, teilrandomisierter Stimulationsblock und darauf folgend neun Nachmessungen.

\subsection{1 tDCS $_{\text {anodal }}$}

Die Gleichstromstimulation erfolgte mit Hilfe eines batteriebetriebenen Hirnstimulators (DC-Stimulator Plus, NeuroConn GmbH, Ilmenau, Deutschland), welcher eine maximale Stromstärke von $5 \mathrm{~mA}$ liefert. Der Gleichstrom floss kontinuierlich mit einer Stromstärke von $1000 \mu \mathrm{A}$ für zehn Minuten mit einer Stromdichte von 62,5 $\mu \mathrm{A} / \mathrm{cm} 2$ von der differenten Elektrode über dem Repräsentationsareal des FDI zur indifferenten Elektrode über der Orbita (anodale tDCS). Dies entspricht den Sicherheitsrichtlinien, welche für die tDCS entwickelt wurden (Nitsche et al. 2003). Innerhalb der ersten acht Sekunden wurde die Stromstärke rampenförmig auf den Zielwert erhöht und in den letzten acht Sekunden wieder gleichmäßig auf Null abgesenkt. Die Stimulation wurde von den meisten Probanden als leichtes Kribbeln unter der Stimulationselektrode für wenige Minuten wahrgenommen.

\subsection{2 iTBS}

Die intermittierende Thetaburststimulation (iTBS) erfolgte mittels eines Magstim Super Rapid Stimulators und der zuvor beschriebenen Magstim-Double-70-mm-Spule. Die Thetaburststimulation wurde sieben Minuten nach Aufzeichnung der Baseline begonnen, um den gleichen Zeitraum zwischen Baseline und erster Nachmessung 
analog zu den anderen Stimulationsarten zu gewährleisten. Den Probanden wurden je eine Dreiersalve Stimuli (engl. train) mit einer Frequenz von $50 \mathrm{~Hz}$ (Interstimulusintervall $20 \mathrm{~ms}$, ISI) alle $200 \mathrm{~ms}$ (5 Hz inter-train interval, ITI) bei 80\% AMT verabreicht (siehe Abbildung 1). Eine TBS-Serie, auch Block genannt, dauerte zwei Sekunden, enthielt folglich zehn Dreiersalven, und wiederholte sich alle zehn Sekunden (engl. $10 \mathrm{~s}$ interblock interval, |BI). Insgesamt wurden mit diesem Protokoll in 190 Sekunden intermittierend 600 Stimulationspulse abgegeben (Huang et al. 2005).

\subsection{3 tRNS}

Die transkranielle Rauschstromstimulation (engl. transcranial random noise stimulation, tRNS) erfolgte ebenfalls mittels einer mikroprozessorgesteuerten Konstantstromquelle (DC-Stimulator Plus, NeuroConn $\mathrm{GmbH}$, IImenau, Deutschland) angelehnt an das Protokoll von Terney et al. (2008). Der Hirnstimulator generierte im Programm "noise“ ein normalverteiltes weißes Rauschen (Abtastrate $1280 \mathrm{sps}$ ). Das Signal ist normalverteilt, da alle Frequenzen bis zur Hälfte der möglichen Wechselrate $(640 \mathrm{~Hz})$ zu gleichen Teilen im Signal enthalten sind. Der Begriff weißes Rauschen bezieht sich auf die spektrale Leistungsdichte des erzeugten Wechselstroms. Die Rauschwerte sind demnach voneinander unabhängig und folgen bei der Betrachtung über ein längeres Zeitintervall idealerweise einer Gauß'schen-Glockenkurve. Des Weiteren ist der angebrachte Strom bipolar und mittelwertfrei. Die hierbei verwendete Stromstärke von $1000 \mu \mathrm{A}$ besagt, das $99 \%$ aller generierten Strompegel zwischen +500 $\mu \mathrm{A}$ und $-500 \mu \mathrm{A}$ lagen. Nur ein Prozent der Strompegel waren größer als $\pm 500 \mu \mathrm{A}$ und die maximal aufgetretenen Stromstärken lagen bei $\pm 600 \mu \mathrm{A}$. (siehe Abbildung 6 ). Dieser randomisierte Strom wurde ebenso wie die tDCS und die Sham-Stimulation für zehn Minuten appliziert. 

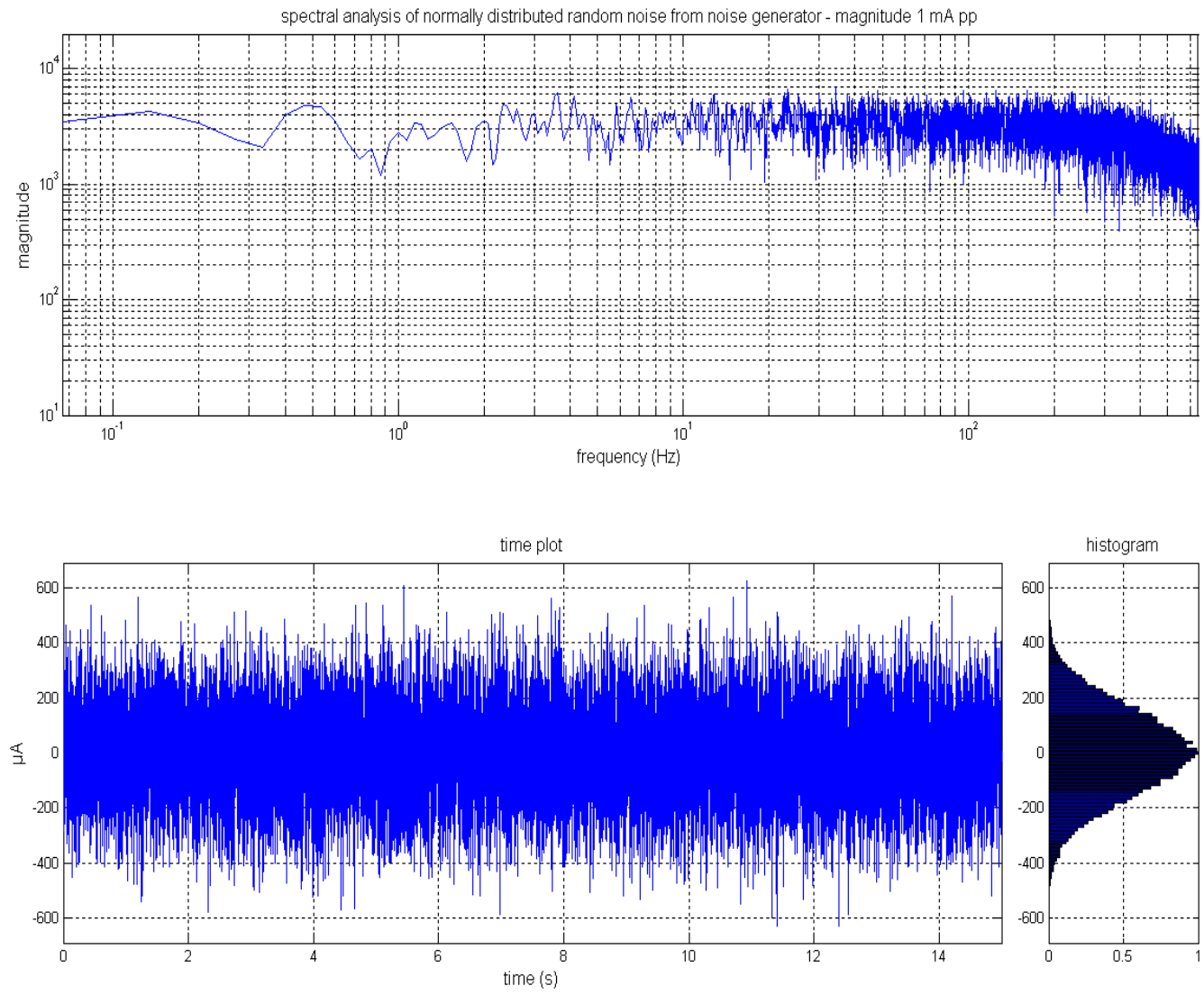

\section{Abbildung 6: Ausgangssignal des DC-Stimulator PLUS}

Die Abbildung zeigt die Frequenzverteilung des Signals, den zeitlichen Verlauf und ein Histogramm. Das Signal ist computergeneriert. Die Amplitude von $1 \mathrm{~mA} \mathrm{pp}$ bedeutet, dass $99 \%$ aller generierten Strompegel zwischen $+500 \mu \mathrm{A}$ und $-500 \mu \mathrm{A}$ liegen.

\subsubsection{Sham-Stimulation}

Um die Sham-Stimulation so realistisch wie möglich wirken zu lassen, wurde hier Gleichstrom für acht Sekunden rampenförmig auf $1 \mathrm{mV}$ aufgebaut und dann wieder ausgeschaltet. Dadurch verspürten die meisten Probanden ein leichtes Kribbeln unter den Elektroden und konnten eine echte Stimulation nicht von einer Sham-Stimulation unterscheiden. Die Sham-Stimulation dauerte zehn Minuten. 


\subsection{Datenauswertung}

Begonnen wurde mit einer deskriptiven Auswertung der erzeugten MEPs. Die durch die Software "Signal" dargstellten Kurven wurden durch das Programm „NuCursor" weiter analysiert. In einem vom Untersucher vorgegeben Intervall errechnete es die Differenz zwischen den Werten des oberen und des unteren Umschlagpunktes (Peak-to-Peak-Amplitude). Pro Messung entstanden so je 40 Werte, aus denen separat für jede Messung das arithmetische Mittel und die Standardabweichung bestimmt wurden (für die Baseline und neun Nachmessungen zu den oben beschriebenen Zeitpunkten). Im gleichen Arbeitsschritt konnten auch artifizielle Werte entfernt werden, die unter anderem durch willkürliche Bewegung des Probanden entstanden waren. Die errechneten MEP-Mittelwerte der Nachmessungen wurden gegenüber der Baseline nicht standardisiert.

\subsubsection{Statistische Auswertung}

Die statistische Auswertung erfolgte mit dem Programm Statistica® (StatSoft Europe $\mathrm{GmbH}$, Hamburg, Deutschland). Dazu wurden mehrere Varianzanalysen für Untersuchungen mit Messwiederholungen (ANOVAs) durchgeführt. Zuerst untersuchten wir die Effektivität der Stimulationen gegenüber der Sham-Stimulation. Dazu verwendeten wir eine ANOVA mit einem Faktor "Stimulationsart" (tDCS vs. Sham; iTBS vs sham; tRNS vs. Sham) und „Messzeitpunkt" (neun Ebenen: 0 - 90 min). Wenn sich ein Haupteffekt für den Faktor "Stimulationsart" oder "Messzeitpunkt" oder eine Interaktion zwischen „Stimulationsart" und „Messzeitpunkt“ zeigten, wurde ein FischerLSD-Test durchgeführt. Ein $p-$ Wert von $<.05$ wurde als signifikant für alle statistischen Untersuchungen gewertet.

Zum Vergleich der nicht-normalisierten MEP-Amplituden der einzelnen Nachmessungen zu der jeweiligen Baseline vor der Stimulation wurde ein Students tTest durchgeführt. 


\section{Ergebnisse}

\subsection{Deskriptive Statistik}

Tabelle 1 zeigt zusammenfassend die durchschnittliche Erhöhung der MEPAmplituden und deren Standardabweichung nach der Stimulation. Den größten Betrag weist dabei die Rauschstromstimulation auf, ohne sich jedoch bei weitergehender Betrachtung signifikant von der tDCSanodal und iTBS zu unterscheiden.

Tabelle 1: Durchschnittliche MEP-Aplituden (MW; $\mathrm{n}=12$ ) zu den einzelnen Messzeitpunkten und deren Standardfehler (SEM).

\begin{tabular}{|c|c|c|c|c|c|c|c|c|}
\hline & \multicolumn{2}{|c|}{ sham } & \multicolumn{2}{|c|}{ tDCS $_{\text {anodal }}$} & \multicolumn{2}{|c|}{ iTBS } & \multicolumn{2}{|c|}{ tRNS } \\
\hline & $M W$ & SEM & $M W$ & SEM & $M W$ & SEM & $M W$ & SEM \\
\hline Base & .98 & \pm .02 & 1.00 & \pm .02 & 1,01 & \pm .02 & 0,95 & \pm .02 \\
\hline $0 \mathrm{~min}$ & .91 & \pm .05 & 1.17 & \pm .08 & 1,19 & \pm .07 & 1,29 & \pm .12 \\
\hline $5 \mathrm{~min}$ & .96 & \pm .04 & 1.19 & \pm .08 & 1,29 & \pm .12 & 1,33 & \pm .14 \\
\hline $10 \mathrm{~min}$ & .97 & \pm .05 & 1.22 & \pm .06 & 1,36 & \pm .12 & 1,26 & \pm .15 \\
\hline $20 \mathrm{~min}$ & .96 & \pm .04 & 1.25 & \pm .12 & 1,28 & \pm .06 & 1,18 & \pm .1 \\
\hline $30 \mathrm{~min}$ & .93 & \pm .03 & 1.20 & \pm .06 & 1,40 & \pm .08 & 1,16 & \pm .15 \\
\hline $40 \mathrm{~min}$ & .93 & \pm .04 & 1.20 & \pm .06 & 1,31 & \pm .08 & 1,16 & \pm .16 \\
\hline $50 \mathrm{~min}$ & .98 & \pm .04 & 1.18 & \pm .05 & 1,33 & \pm .07 & 1,07 & \pm .14 \\
\hline $60 \mathrm{~min}$ & 1.00 & \pm .04 & 1.23 & \pm .07 & 1,35 & \pm .06 & 1,09 & \pm .15 \\
\hline $90 \mathrm{~min}$ & .93 & \pm .03 & 1.19 & \pm .1 & 0,96 & \pm .04 & 1,03 & \pm .05 \\
\hline Gesamt & .955 & \pm .02 & 1.18 & \pm .03 & 1.15 & \pm .05 & 1.29 & \pm 1.0 \\
\hline
\end{tabular}

Anmerkung: Alle Werte in $\mathrm{mV}$ und auf zwei Stellen gerundet. $\mathrm{tDCS}_{\text {anodal }}=$ anodale transkranielle Gleichstromstimulation; tRNS = transkranielle Rauschstromstimulation; iTBS = intermittierende Thetaburststimulation; sham = Sham-Stimulation; Base = Messung vor der Stimulation

\subsection{Intensität}

Abbildung 7 zeigt die Mittelwerte und die Standardabweichung der Stimulatorintensitäten, die bei den einzelnen Stimulationen zur Anwendung kamen. Es ist erkennbar, dass sich der durchschnittliche Stimulatoroutput bei den einzelnen Stimulationsarten kaum unterscheidet und so keinen Einfluss auf das Ergebnis der Stimulation nehmen konnte. 


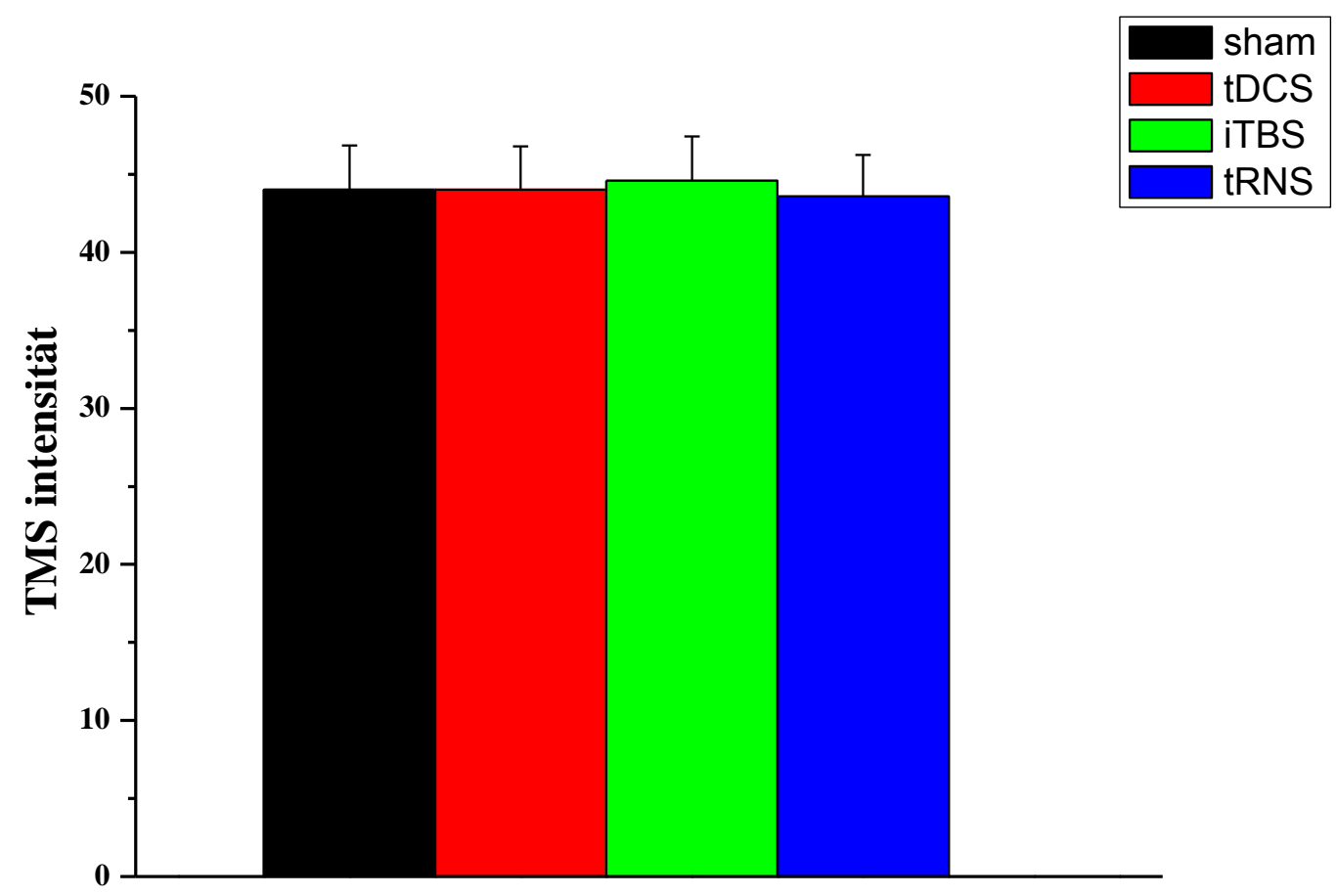

\section{Abbildung 7: Stimulatorintensität}

Output des Magstim 200 in Prozent (\%) seiner vollen Leistungsstärke im Mittel der jeweiligen Stimulationsart. Es gibt im Durchschnitt kaum Unterschiede zwischen den einzelnen Messungen. Die Ausziehungen der senkrechten Balken bilden die Standardabweichung ab.

\subsection{Varianzanalysen}

\subsection{1 tDCS $_{\text {anodal }}$}

Die zehnminütige Stimulation mit $1 \mathrm{~mA}$ Intensität über dem Repräsentationsareal des FDI erhöhte die kortikale Exzitabilität um 21\% (Abbildung 8). Durch ANOVAs mit Messwiederholungen konnte ein signifikanter Haupteffekt der tDCS für "Stimulation“, F1.11 $=32.5, p=.0001$, nachgewiesen werden. Der Faktor "Messzeitpunkt" verfehlte knapp das Signifikanzniveau, F9.99 $=0,83, p=.6$. Es zeigte sich keine signifikante Interaktion zwischen „Stimulation“ und „Messzeitpunkt“, F9.99 = .96, $p=.5$. Signifikant erhöhte MEPs nach tDCS wurden entsprechend der post-hoc-Analyse zwischen 0 min und $90 \mathrm{~min}$, verglichen mit den Zeitpunkten der Sham-Stimulation gemessen, alle ps < .0001. Den Student's t-Test führten wir zum Vergleich der MEP-Amplituden nach Stimulationsende $(0,5,10,20$, bis $90 \mathrm{~min})$ mit den MEP-Amplituden der Baseline durch. 
Die Analyse mit dem Student's t-test zeigte eine signifikante Erhöhung der MEPAmplituden nach tDCS zu den Zeitpunkten fünf min bis 90 min $(p<.05)$ verglichen mit der Baseline.

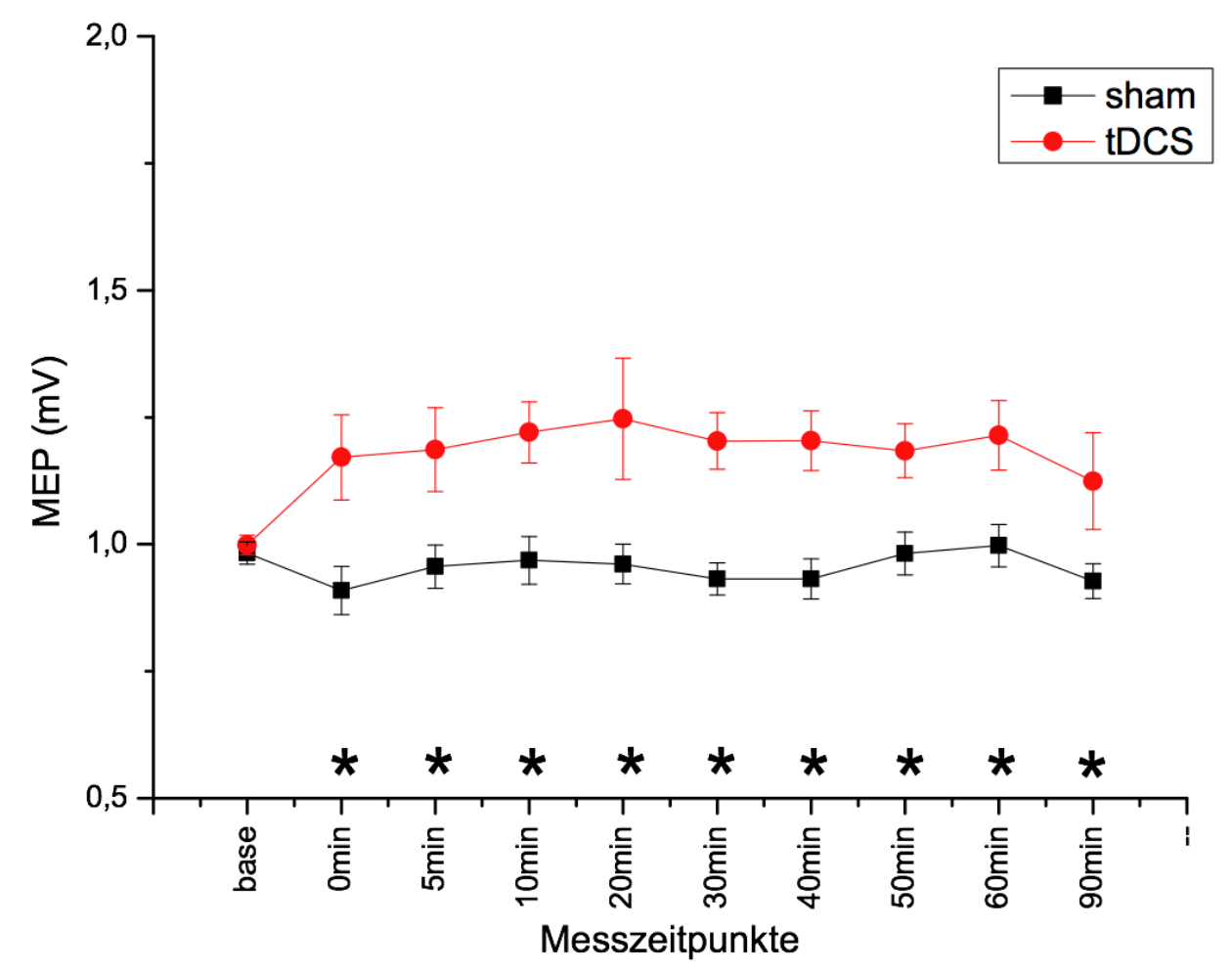

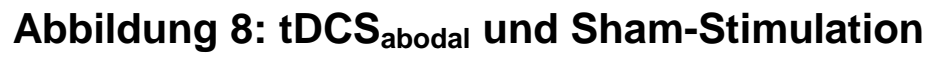

Grafische Darstellung der Veränderung der MEP-Amplituden nach zehn Minuten tDCS ${ }_{\text {anodal }}$. Daten gemittelt aus je 12 Einzelmessungen pro Messzeitpunkt an zwölf Probanden. Die Sternchen markieren die Zeitpunkte nach der Stimulation mit signifikant erhöhten MEP-Amplituden der tDCS ${ }_{\text {anodal }}$ gegenüber der Sham-Stimulation. Die senkrechten Balken bilden den Standardfehler ab.

\subsection{2 iTBS}

Die dreiminütige Stimulation mit insgesamt 600 Pulsen über dem Repräsentationsareal des FDI erhöhte die kortikale Exzitabilität um 24\% (Abbildung 9). ANOVAs mit Messwiederholungen zeigten einen signifikanten Haupteffekt für "Stimulation“, F1.11 = 17,9 p =.001, und „Messzeitpunkt“, F9.99 = 2,57, p = .01. Auch die Interaktion zwischen „Stimulation“ und „Messzeitpunkt“ war signifikant, F9.99 = 2,87, $p=.005$. Die post-hoc-Analysen ergaben signifikant erhöhte Amplituden für die 
Thetaburststimulation zwischen 0 Minuten bis 40 Minuten verglichen mit den gleichen Zeitpunkten der Sham-Stimulation, ps <.006. Die Analyse mit dem Student's t-test zeigte eine signifikante Erhöhung der MEP-Amplituden nach iTBS zu den Zeitpunkten 0 min bis 40 min, alle $p s<.005$, und 60 min, $p=.03$, verglichen mit der Baseline.

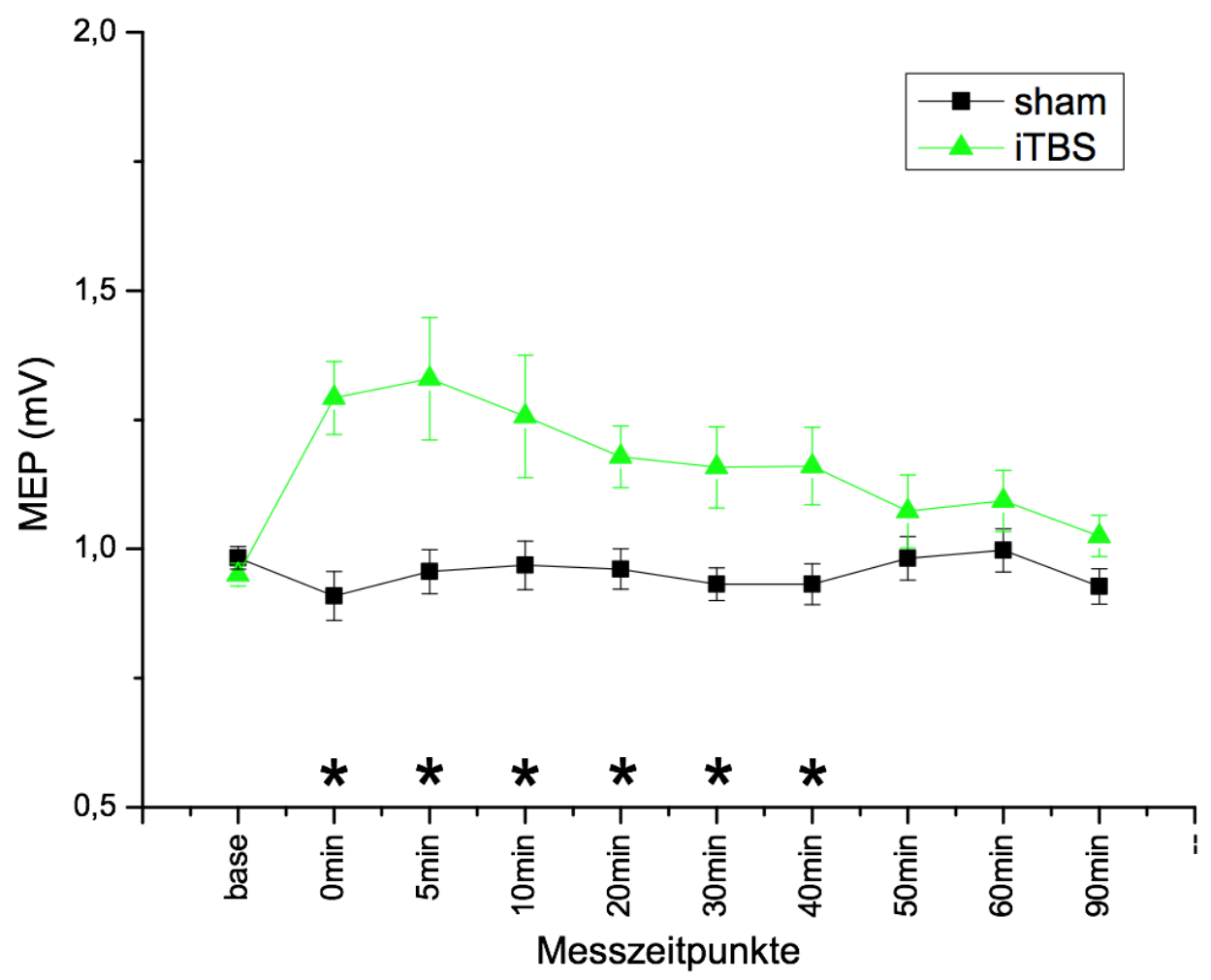

\section{Abbildung 9: iTBS und Sham-Stimulation}

Grafische Darstellung der Veränderung der MEP-Amplituden nach dreiminütiger iTBS (600 Pulse). Daten gemittelt aus je zwölf Einzelmessungen pro Messzeitpunkt an zwölf Probanden. Die Sternchen markieren die Zeitpunkte nach der Stimulation mit signifikanten unterschiedlichen MEP-Amplituden zwischen iTBS und Sham-Stimulation. Die senkrechten Balken bilden den Standardfehler ab.

\subsection{3 tRNS}

Die zehnminütige Stimulation mit $1 \mathrm{~mA}$ Intensität über dem Repräsentationsareal des FDI erhöhte die kortikale Exzitabilität um 33\% (Abbildung 10). ANOVAs mit Messwiederholungen zeigten einen signifikanten Haupteffekt für „Stimulation“, F1.11 = $10,696, p=.007$, und „Messzeitpunkt“, F9.99 $=3,13, p=.002$. Auch die Interaktion zwischen „Stimulation“ und "Messzeitpunkt“ war signifikant, F9.99 $=2,99, p=.003$. Die 
post-hoc-Analysen ergaben signifikant erhöhte Amplituden für die Rauschstromstimulation zwischen $0 \mathrm{~min}$ und $60 \mathrm{~min}$ verglichen mit den gleichen Zeitpunkten der Sham-Stimulation, ps < .005. Die Analyse mit dem Student's t-test zeigte eine signifikante Erhöhung der MEP-Amplituden nach tRNS zu den Zeitpunkten fünf bis 60 Minuten, alle ps > .005, verglichen mit der Baseline.

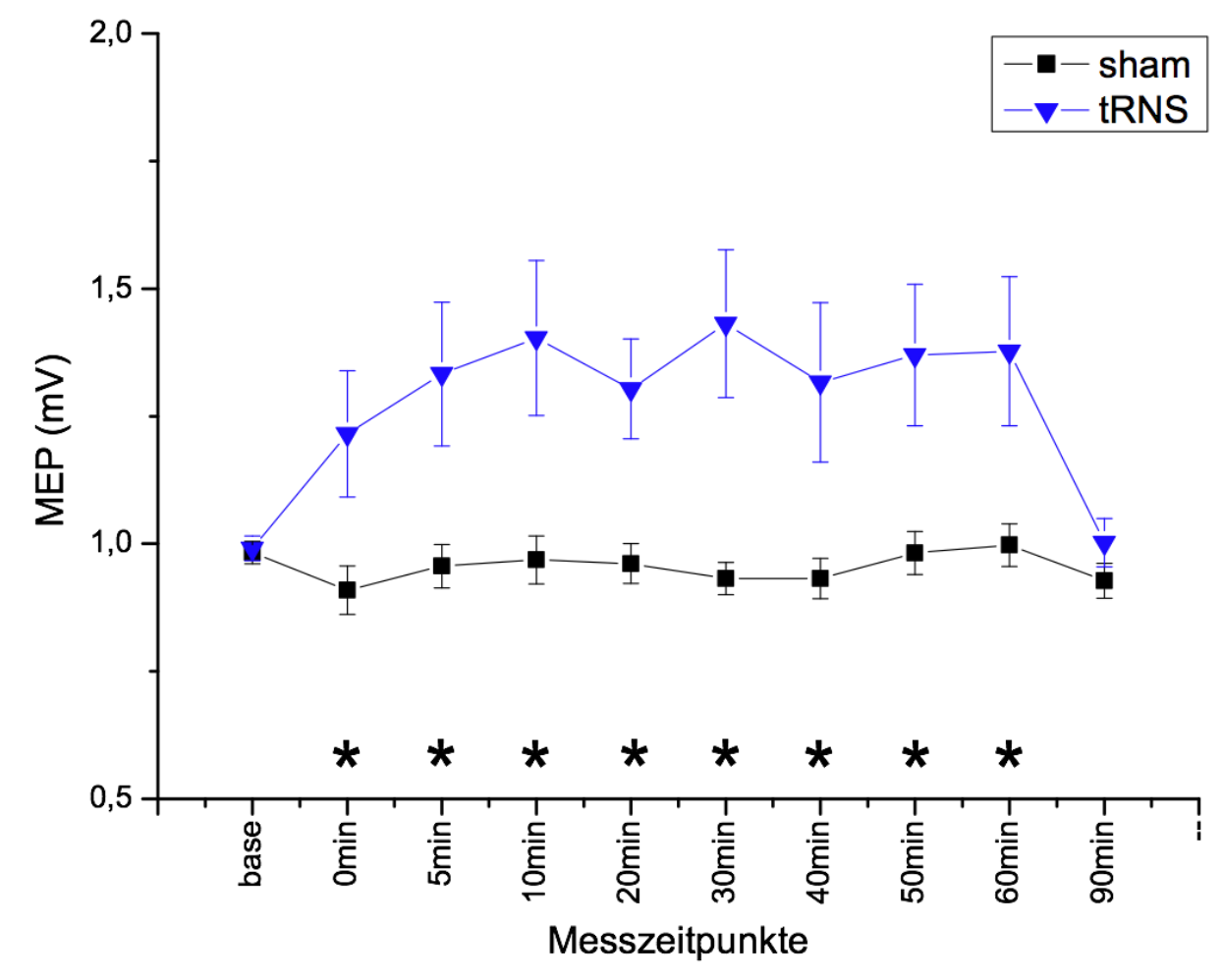

\section{Abbildung 10: tRNS und Sham-Stimulation}

Grafische Darstellung der Veränderung der MEP-Amplituden nach zehnminütiger tRNS. Die Sternchen markieren die Zeitpunkte nach der Stimulation mit signifikanten Unterschieden zwischen tRNS und Sham-Stimulation. Daten gemittelt aus je zwölf Einzelmessungen pro Messzeitpunkt an zwölf Probanden. Die senkrechten Balken bilden den Standardfehler ab. 


\subsubsection{Vergleich der Stimulationsarten untereinander}

Sämtliche Haupteffekte und Interaktionen („Stimulation“ und „Messzeitpunkt“) der einzelnen Stimulationen (1. tRNS verglichen mit tDCSanodal, 2. tRNS verglichen mit iTBS und 3. tDCSanodal verglichen mit iTBS) unterschieden sich nicht signifikant (siehe Tabelle 2). Alle vier Stimulationsarten gleichzeitig verglichen, ergaben einen Effekt von $\mathrm{F} 3,33=6.44, \mathrm{p}<.05$. Die anschließenden post-hoc-Analysen zeigten, dass die Amplituden der Sham-Stimulation signifikant kleiner waren im Vergleich zu tDCSanodal, $p=.001$, iTBS, $p<.01$ und tRNS, $p<.05$ (siehe Tabelle 2).

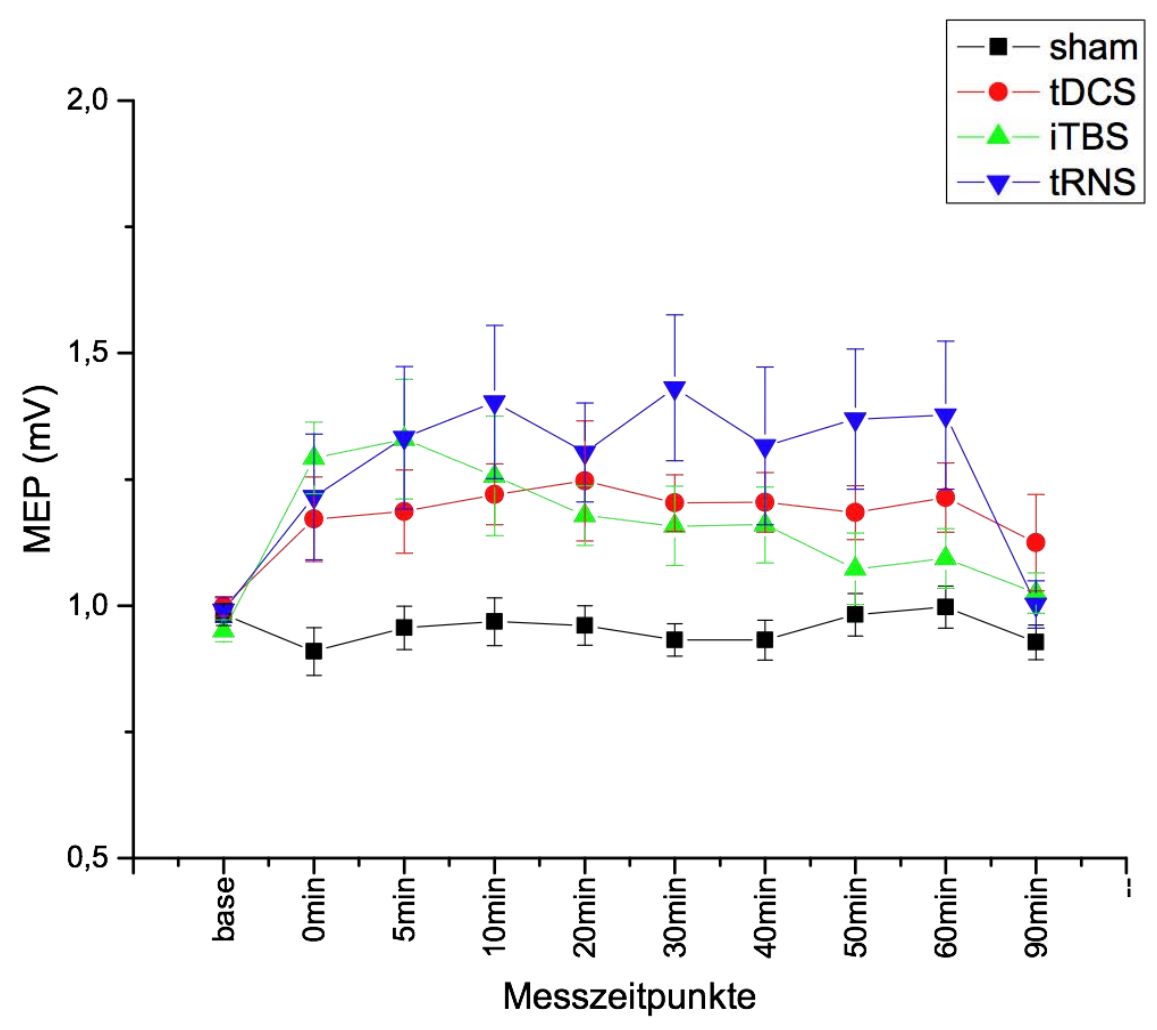

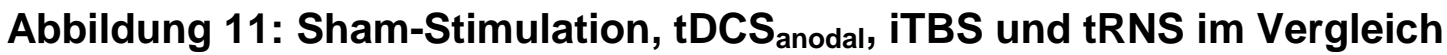

Grafische Darstellung aller Stimulationsarten. Es gibt keine signifikanten Unterschiede zwischen tRNS, tDCS ${ }_{\text {anodal }}$ und iTBS. 
Tabelle 2: Vergleich Amplituden (mV) aller 4 Stimulationsarten

\begin{tabular}{|c|c|c|c|c|c|c|}
\hline \multirow{2}{*}{$\begin{array}{l}\text { Stimulation } \\
\text { (I) }\end{array}$} & \multirow{2}{*}{$\begin{array}{l}\text { Stimulation } \\
\text { (J) }\end{array}$} & \multirow{2}{*}{$\begin{array}{c}\text { Mittlere } \\
\text { Differenz } \\
(\mathrm{I}-\mathrm{J})\end{array}$} & \multirow{2}{*}{$\begin{array}{c}\text { Standard- } \\
\text { fehler }\end{array}$} & \multirow[b]{2}{*}{ Sig. $^{a}$} & \multicolumn{2}{|c|}{$\begin{array}{l}\text { 95\% Konfidenzintervall } \\
\text { für die Differenz }{ }^{\mathrm{a}}\end{array}$} \\
\hline & & & & & Untergrenze & Obergrenze \\
\hline sham & $\begin{array}{l}\text { tDCS } \\
\text { iTBS } \\
\text { tRNS }\end{array}$ & $\begin{array}{l}.221^{*} \\
-.197^{*} \\
-.319^{*}\end{array}$ & $\begin{array}{l}.039 \\
.047 \\
.098\end{array}$ & \begin{tabular}{l|}
.001 \\
.008 \\
.045
\end{tabular} & $\begin{array}{l}-.345 \\
-.346 \\
-.633\end{array}$ & $\begin{array}{l}-.096 \\
-.048 \\
-.006\end{array}$ \\
\hline tDCS ${ }_{\text {anodal }}$ & $\begin{array}{l}\text { sham } \\
\text { iTBS } \\
\text { tRNS }\end{array}$ & $\begin{array}{l}.221^{*} \\
.024 \\
-.099\end{array}$ & $\begin{array}{l}.039 \\
.047 \\
.092\end{array}$ & $\begin{array}{l}.001 \\
1.000 \\
1.000\end{array}$ & $\begin{array}{r}.096 \\
-.128 \\
-.393\end{array}$ & $\begin{array}{l}.345 \\
.176 \\
.195\end{array}$ \\
\hline iTBS & $\begin{array}{l}\text { sham } \\
\text { tDCS } \\
\text { tRnodal } \\
\text { tRNS }\end{array}$ & $\begin{array}{l}.197^{*} \\
-.024 \\
-.123\end{array}$ & $\begin{array}{l}.047 \\
.047 \\
.109\end{array}$ & $\begin{array}{l}.008 \\
1.000 \\
1.000\end{array}$ & $\begin{array}{c}.048 \\
-.176 \\
-.471\end{array}$ & $\begin{array}{l}.346 \\
.128 \\
.226\end{array}$ \\
\hline tRNS & $\begin{array}{l}\text { sham } \\
\text { tDCS } \\
\text { iTBSodal } \\
\text { iTBS }\end{array}$ & $\begin{array}{l}.319^{*} \\
.099 \\
.123\end{array}$ & $\begin{array}{l}.098 \\
.092 \\
.109\end{array}$ & $\begin{array}{l}.045 \\
1.000 \\
1.000\end{array}$ & $\begin{array}{r}.006 \\
-.195 \\
-.226\end{array}$ & $\begin{array}{l}.633 \\
.393 \\
.471\end{array}$ \\
\hline
\end{tabular}

Anmerkungen: Basiert auf den geschätzten Randmitteln. Alle Werte auf zwei Stellen gerundet.

*. Die mittlere Differenz ist auf dem ,05-Niveau signifikant.

a. Anpassung für Mehrfachvergleiche: Bonferroni. Sig. = Signifikanzniveau. 


\section{Diskussion}

In der aktuellen Forschung wurde eine Reihe von neuen oder modulierten Techniken zur transkraniellen Hirnstimulation eingeführt; darunter die Gleichstromstimulation mit unterschiedlichsten Protokollen (für eine Übersicht dazu siehe Nitsche et al. 2008), die Thetaburststimulation (Huang et al. 2005), die Wechselstromstimulation (Antal et al. 2008, Kanai et al. 2008) und die Rauschstromstimulation (Terney et al. 2008). Alle Stimulationsarten konnten ihre Effektivität in Bezug auf die Modulation der kortikalen Exzitabilität beweisen. Jedoch ist bislang nicht gezeigt worden, ob jeder Proband oder Patient auf alle diese neuen Stimulationstechniken reagiert. Aus diesem Grund wurden in der vorliegenden Untersuchung zwei etablierte Stimulationstechniken (tDCSanodal und iTBS) und eine neuere Methode (tRNS) am gleichen Probandenkollektiv getestet.

Die Ergebnisse der vorliegenden Arbeit zeigten, dass alle drei Stimulationsarten (tDCSanodal, iTBS und tRNS) die Exzitabilität des motorischen Kortex nachhaltig erhöhen können, wenn auch mit jeweils unterschiedlicher Charakteristik in der Neuromodulation. Während die tDCSanodal einen moderaten und stabilen Anstieg der Exzitabilität über die gesamte Zeit der Nachmessung (90 min) erzeugte (Abbildung 8), ist dies bei der iTBS nur für 40 Min (Abbildung 9) und bei der tRNS für bis zu 60 Min (Abbildung 10) der Fall. Damit konnten die bisherigen Studienergebnisse zu den gewählten Stimulationen bestätigt werden (unter anderem Huang et al. 2005, Nitsche et al. 2008, Terney et al. 2008). Die Intensität des Magnetstimulators lag, wie gezeigt, (Abbildung 7) bei allen Nachmessungen zu jeder Stimulation bei ungefähr $44 \%$. Hieraus kann man schließen, dass die TMS-Stimulationsintensität auf die gefundenen Ergebnisse keinen Einfluss hat. Des Weiteren konnten keine Unterschiede in der Höhe der Exzitabilitätserhöhung zwischen tRNS und den etablierten Stimulationsarten gefunden werden. Die Rauschstromstimulation lag dem Betrag der Neuromodulation nach in dem Bereich, den auch die anderen zwei Stimulationen vorgegeben haben. Die Unterschiede im zeitlichen Verlauf der beobachteten Amplitudenverstärkung nach den jeweiligen Stimulationen lassen auf verschiedene Mechanismen schließen, welche die gemessenen Phänomene erklären können. 
Während der tDCS kommt es im stimulierten Kortexareal zu einer Verschiebung des Ruhemembranpotentials, jedoch ohne dass direkt Aktionspotentiale ausgelöst werden. Diese tonische Verschiebung des Membranpotentials moduliert die Erregbarkeit der kortikalen Neurone und beeinflusst ihre Spontanaktivität. Die grundlegenden tierexperimentellen Arbeiten, welche zu diesen Erkenntnissen führten, stammen von Bindman et al. (1964) und Purpura und McMurtry (1965). Hier wurde die Abhängigkeit der Spontanentladungsrate von Neuronen von kortikal appliziertem Gleichstrom an anästhesierten Ratten untersucht. Die anodale Stimulation führte zu einer unterschwelligen Depolarisation und Erregungssteigerung des Kortex. Die kathodale Stimulation führte hingegen zur Hyperpolarisierung und zur Abnahme der Spontanaktivität der stimulierten Neurone. Des Weiteren konnte gezeigt werden, dass die Dauer der Stimulation sich für die Dauer der Nacheffekte (Bindman et al. 1964) verantwortlich zeigt. Ein fünf Minuten applizierter Gleichstrom führte nur zu kurzen Nacheffekten, während längere Polarisierung mit Gleichstrom die Effekte auf die Exzitabilität bis zu mehreren Stunden nach Beendigung der Stimulation anhalten ließ. Dies lässt eine gesteigerte synaptische Effizienz im Sinne einer Langzeitpotenzierung (long-term potentiation, LTP) bei längerer Stimulationsdauer vermuten. Dafür sprechen auch die biochemischen Veränderungen, welche bei längeren anodalen Gleichstromstimulationen auftreten und gleichzeitig charakteristisch für LTP sind. Dazu gehören ein durch Noradrenalin ausgelöster Anstieg des intrazellulären cAMP-Spiegels sowie ein Anstieg des intrazellulären Kalziums (Hattori et al. 1990, Islam et al. 1995). Liebetanz et al. (2002) bewiesen die Abhängigkeit der Gleichstrom-Nacheffekte von NMDA-Rezeptor-Aktivierung. Sie konnten die Effekte von anodaler und kathodaler tDCS, welche nach der Stimulation auftreten, durch den nichtkompetitiven NMDARezeptor-Antagonisten Dextromethorphan vollständig unterdrücken.

Die Wirkung der lang anhaltenden Konditionierungseffekte nach Ende der Thetaburststimulation beruht auch hier auf der sogenannten Langzeitpotenzierung (long term potentiation, LTP). Dabei gelten N-Methyl-D-Aspartat-Rezeptoren (NMDA) als die wichtigen Rezeptoren für die LTP-Induktion (Toyoda et al. 2006), indem sie die Effektivität synaptischer Übertragung verbessern. Huang et al. (2007) konnten die Abhängigkeit der TBS von NMDA-Rezeptoren belegen, indem sie die TBS-Wirkung auf den Motorkortex mit einem NMDA-Antagonist (Memantine) blockierten. 
Ein möglicher Mechanismus für die Wirkung der tRNS könnte die Aktivierung von Natrium-Kanälen mittels Na-Gleichrichtung durch hochfrequente Stromstimulation der unterschwellig erregten Membran sein (Bromm 1968). Zuerst bewirkt die Stimulation eine Depolarisation der Zellmembran. Dies führt dann zu einem Einstrom von Na+-lonen entlang des Konzentrationsgradienten. Dieser $\mathrm{Na}+$-Einstrom verstärkt die Membrandepolarisation. Ist der Natrium-Einstrom nicht ausreichend, kommt es nicht zu einer regenerativen Depolarisation und folglich auch nicht zu einem Aktionspotential. Da die Repolarisation der Zellmembran verglichen mit der Depolarisation länger dauert,

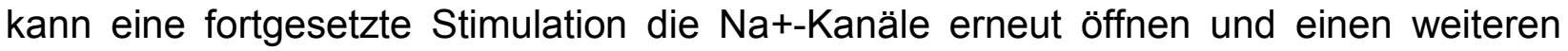
$\mathrm{Na}+$-Einstrom hervorrufen. Infolgedessen wird die Zellmembran weiter depolarisiert und verstärkt den Effekt der vorangegangenen Depolarisation. Man kann annehmen, dass eine Membrandepolarisation, die länger als drei Minuten dauert, ähnlich der tDCS zu LTP-artigen Mechanismen an den Synapsen der stimulierten Neuronen führt (Terney et al. 2008). Terney et al. (2008) vermuteten, dass die multiple Öffnung von Natriumkanälen mit dem oben beschrieben Mechanismus zu einem substantiell höheren Effekt auf die kortikale Exzitabilität führen müsse, als die einmalige Öffnung derselben mit der Gleichstromkomponente der tDCS. Diese Vermutung ist nach den Ergebnissen dieser Studie nicht zutreffend.

Die dargestellten Erkenntnisse und Vermutungen sind aktuell Gegenstand intensiver Forschung. Gleichzeitig konnte in der vorliegenden Arbeit gezeigt werden, dass unterschiedliche Mechanismen, manche bekannt, manche noch unbekannt, zu ähnlichen Effekten führen. Die tRNS ist nach den vorliegenden Ergebnissen der iTBS und der tDCS im Betrag der Exzitabilitätserhöhung nicht überlegen. Die vergleichbaren Effekte der Neuromodulation im direkten Vergleich lassen jedoch die Vermutung zu, dass klinische Ergebnisse, die zum Beispiel mit der tDCS erzielt wurden (siehe Kapitel 1.1.2), auch mit anderen Stimulationsarten erfolgreich wiederholt oder sogar verbessert werden können. Dabei kann man sich die Vorteile der einzelnen Stimulationsarten zu nutze machen.

Ein möglicher Vorteil der Gleichstromstimulation könnte die Länge der erzeugten Konditionierungseffekte sein. Nitsche und Paulus (2000) beschreiben die Erhöhung der MEP-Amplituden nach elf min tDCS mit $1 \mathrm{~mA}$ für bis zu 60 min nach Stimulationsende. 
Wir konnten in der vorliegenden Studie eine Exzitabilitätserhöhung von bis zu 90 min nach zehn min tDCS feststellen.

Vorteilhaft an der Rauschstromstimulation ist, dass sie von den Probanden oder Patienten so gut wie nicht wahrgenommen wird (Terney et al. 2008) und sich deshalb wesentlich besser für eine Verblindung im Rahmen von klinischen Studien eignet als beispielsweise die Thetaburststimulation und die Gleichstromstimulation.

Neueste Studien zur Wirksamkeit von Neuroplastizität-induzierenden Stimulationen in Abhängigkeit vom Genotyp des Patienten geben neue Hinweise zur Verwendung der einzelnen Stimulationsarten. Untersucht wurde die Effektivität von tDCS, iTBS und tRNS bei Trägern unterschiedlicher Allelkombinationen des brainderived neurotrophic factor (BDNF). Dieser Faktor gehört zur Familie der Neurotrophine und regelt unter anderem das Überleben und die Differenzierung von Neuronen im zentralen Nervensystem. Des Weiteren ist er an der aktivitätsabhängigen synaptischen Plastizität im heranwachsenden und adulten Gehirn beteiligt (Cowansage et al. 2009). Es wurde festgestellt, dass heterozygote Träger besser auf Gleichstromstimulation reagieren, wohingegen nur bei homozygoten Merkmalsträgern eine Exzitabilitätssteigerung mit iTBS ausgelöst werden konnte (Antal et al. 2010). Außerdem konnten die Autoren zeigen, dass die Effekte der Rauschstromstimulation nicht durch den Genotyp des Probanden beeinflusst werden. Hier könnte sich die tRNS in Zukunft als vorteilhaft erweisen, wenn sie unabhängig von genetischen Faktoren stabile Neuromodulationen hervorrufen kann. Dazu sind allerdings weiterführende Studien nötig.

Ein weiterer neuer Ansatz ist es, durch transkranielle Stimulationen mit hirneigenen Oszillationsmustern zu interferieren. Seit einigen Jahren sind hirneigene Synchronisation und Oszillation in den Fokus der Neurowissenschaften gerückt (für einen Überblick dazu siehe: Buzsáki und Draguhn 2004) und man macht sie für eine Reihe von wichtigen höheren Hirnfunktionen verantwortlich. So sind Oszillationen zum Beispiel unverzichtbar für die Funktion unseres Kurzzeitgedächtnisses (Lisman und Idiart 1995) und darüber hinaus ist die suffiziente Synchronisation zwischen visuellen und motorischen Kortexarealen mittels Oszillationen entscheidend für eine funktionierende Visomotorik (Roelfsema et al. 1997). Des Weiteren fand Singer (1999) unter anderem heraus, dass neuronale Synchronisation mitverantwortlich für die 
synaptische Effizienz ist. Das Konzept der synaptischen Effizienz im Sinne von Langzeitpotenzierung und Langzeitdepression (LTP und LTD) ist wiederum essentieller Kern der Plastizitätsforschung mit transkraniellen Stimulationsmethoden. Hinzu kommt, dass beispielsweise die Rauschstromstimulation mit ihrer oszillierenden Stimulationscharakteristik, wie in dieser Studie gezeigt, effektive Exzitabilitätsveränderung im Kortex auslösen kann. Diese Oszillation könnte ein möglicher Mechanismus für die Wirkungsweise der Rauschstromstimulation sein, im Gegensatz zur den polarisierenden Stimulationen (z.B. Gleichstromstimulation). Weitere Forschungen mit frequenzgebundenen Stimulationen laufen bereits. So wird derzeit versucht, durch Wechselstromstimulation mit ganz bestimmten Frequenzbändern (80 $\mathrm{Hz}, 140 \mathrm{~Hz}$ und $250 \mathrm{~Hz}$, sog. Ripple-Oszillationen) neuroplastische Modulationen hervorzurufen. Dieses Konzept beruht darauf, hirneigene Oszillationen, welche die synaptische Effizienz verbessern, durch transkranielle Techniken zu simulieren. Hier werden also bestimmte Frequenzen, die in der Rauschstromstimulation enthalten sind, extrahiert und somit „rauschfrei“ dem Gehirn zugeführt. Inwieweit das Vorteile bringt, ist zum heutigen Zeitpunkt noch nicht vorhersehbar.

Dennoch birgt das Konzept der oszillierenden Stimulation weitere Optionen für den Versuch der klinischen Anwendung. Fuentes et al. (2009) nutzten oszillierende Stimulation im Bereich von $300 \mathrm{~Hz}$, angebracht mit epiduralen Elektroden am Hinterhorn des Rückenmarks von Mäusen mit Morbus Parkinson, um deren Fähigkeiten zu Fortbewegung zu verbessern. Damit konnten nicht nur die Bewegungsabläufe an sich verbessert werden, sondern sie stellten auch ein erhöhtes Maß an Bewegungsinitiierung fest. Daraus folgerten sie, dass Gehirnareale, die an motorischen Abläufen beteiligt sind, durch die oszillierende Stimulation des Rückenmarks in die Lage versetzt werden, Bewegungen zu initiieren.

Ein möglicher Mechanismus dazu ist, dass die Stimulation des Rückenmarks bestimmte Erregungszentren im Hirnstamm aktiviert, welche dann zu einer suffizienten Desynchronisation des Kortex und striataler Bahnen führen. Dies ist laut Fanselow et al. (2000) nötig, um freiwillig eine Bewegung zu initiieren. Auch hier ist wieder denkbar, mit transkraniellen Stimulationen diese zur Willkürbewegung erforderliche Desynchronisation des Kortex hervorzurufen oder zu beeinflussen und damit ParkinsonSymptome zu lindern. 


\section{Zusammenfassung}

Die Neuroplastizität und die zugrundeliegenden Mechanismen sind aktuell Gegenstand intensiver Forschung und auf dem Weg, die Ursprünge von Lernen, Gedächtnis und Kognition zu ergründen.

Hierfür gibt es inzwischen eine große Anzahl transkranieller elektrischer und magnetischer Stimulationsverfahren, die auf unterschiedlichste Weise wirken, aber alle das Ziel haben, den Erregungszustand des menschlichen Kortex in der gewünschten Richtung zu beeinflussen. Ziel dieser Studie war es, herauszufinden wie prominente Vertreter der einzelnen Stimulationsgruppen (Gleichstrom, Rauschstrom, repetitive Magnetstimulation) im direkten Vergleich in der Lage sind, die Exzitabilität des menschlichen Kortex zu erhöhen. Des Weiteren sollte untersucht werden, ob die spezielle Charakteristik der einzelnen Stimulationsarten mögliche Hinweise auf eine potentielle klinische Anwendung liefern kann.

In der vorliegenden Studie wurde Gleichstromstimulation (tDCSanodal, $10 \mathrm{~min}, 1$

$\mathrm{mA}$ ), intermittierende Thetaburststimulation (iTBS, 600 Pulse) und die Rauschstromstimulation (tRNS, $10 \mathrm{~min}, 1 \mathrm{~mA}$ ) sowie eine Sham-Stimulation miteinander verglichen. Die einzelnen Stimulationsarten sollten ihre Effektivität gegenüber der ShamStimulation sowie untereinander beweisen. Hierfür wurde das Repräsentationsareal eines kleinen Handmuskels stimuliert und der Erfolg der Stimulation mittels EinzelpulsTMS (transkranielle Magnetstimulation) überprüft. Die Veränderungen der MEPs (motorisch evozierte Potentiale) nach Stimulation gaben Aufschluss über die Effektivität der einzelnen Stimulationsarten. Zwölf Versuchsteilnehmer wurden mit allen vier Techniken an verschiedenen Tagen stimuliert und im Anschluss zu unterschiedlichen Zeitpunkten innerhalb von 90 Minuten nachgemessen. Bei allen drei Stimulationsarten zeigte sich eine Exzitabilitätserhöhung gegenüber der Sham-Stimulation. Der zeitliche Verlauf dieser Effekte unterschied sich zwar von den einzelnen Stimulationsarten, aber der Betrag der Erregungssteigerung des Kortex war bei allen in etwa gleich. Da vermutlich jeder Stimulationsart bei ähnlicher Effektivität ein anderer Wirkmechanismus zu Grunde liegt, ergeben sich daraus auch unterschiedliche Anwendungen im klinischen Setting und damit möglicherweise ein breiter Anwendungsbereich für transkranielle Stimulationen. 


\section{Anhang}

\subsection{Einverständniserklärung}

\section{Einverständniserklärung Proband/innen zur Teilnahme an der Untersuchung: Einfluss von schwachem transkortikal applizierten Gleichstrom und randomisierten Stroms auf die zerebrale Exzitabilität und dessen Modulierbarkeit durch transkranielle \\ Magnetstimulation}

Frau/Herr Dr. hat mir heute anhand der Hinweise auf dem Informationsbogen für Teilnehmerinnen und Teilnehmer an der o.g. Studie die Durchführung der Untersuchung erläutert. Ich habe den Aufklärungstext gelesen und verstanden. Ich hatte die Möglichkeit, Fragen zu stellen, und habe die Antworten verstanden und akzeptiere sie. Alle mich interessierenden Fragen wurden ausreichend beantwortet. Mein Arzt hat mich über die mit der Teilnahme an der Studie verbundenen Risiken und den möglichen Nutzen informiert.

Ich erkläre darüber hinaus, dass ich alle Angaben zur Krankengeschichte wahrheitsgemäß gemacht habe. Mir ist bekannt, dass ich jederzeit ohne Angabe von Gründen die weitere Untersuchung ablehnen bzw. meine Einverständniserklärung widerrufen kann, ohne dass mir daraus Nachteile entstehen. Ferner ist mir bekannt, dass ich auch mein Einverständnis zur Speicherung personenbezogener Daten widerrufen kann, ohne dass mir daraus Nachteile entstehen. Ich bin bereit, an der Studie teilzunehmen.

Ich bin damit einverstanden, dass Daten, welche sich aus der Studie ergeben, ohne Erwähnung meiner Identität anonym veröffentlicht oder an zuständige Behörden weitergeleitet werden. Alle Befunde sind vertraulich und werden unter strikter Einhaltung des Datenschutzes gehandhabt. Zudem dürfen die Untersuchungstermine, mein Name und der Name der Studie in einer abteilungsinternen Datenbank dokumentiert werden. Eine Kopie dieser Erklärung wurde mir überreicht.

Göttingen, den

Name des aufklärenden Arztes (in Druckbuchstaben) und Unterschrift

Name des Patienten (in Druckbuchstaben) und Unterschrift 


\subsection{Probandeninformation}

\section{Information für Teilnehmerinnen und Teilnehmer der Untersuchung: Einfluss von schwachem transkortikal applizierten Gleichstrom und randomisierten Stroms auf die zerebrale Exzitabilität}

Sehr geehrte Teilnehmerin, sehr geehrter Teilnehmer!

Wir möchten Sie bitten, an o.g. wissenschaftlicher Untersuchung teilzunehmen und möchten Ihnen den Ablauf der Studie anhand dieses Informationsbogens erläutern. Sie selbst werden nicht unmittelbar von dieser Studie profitieren, die Ergebnisse ermöglichen es uns jedoch, weiterführende Erkenntnisse über den Einfluss schwachen Wechselstroms auf die zerebrale Erregbarkeit des Gehirns zu gewinnen.

In den Sitzungen werden zwei Schwammelektroden auf Ihrem Kopf angebracht. Über diese Elektroden fließt dann für $10 \mathrm{~min}$ ein schwacher Gleichstrom oder ein schwacher randomosierter Strom. Dieser Strom ist für Sie nicht oder allenfalls sehr geringfügig wahrnehmbar. Dieses nicht invasive (=nicht verletzende) und schmerzfreie Verfahren wurde bereits in mehreren Untersuchungen angewendet und hat sich als risiko- und nebenwirkungsarm erwiesen. Selten und lediglich nach kontinuierlicher Stromapplikation über Stunden traten leichte Kopfschmerzen und Hautreizungen im Bereich der Elektroden auf, die sich relativ schnell vollständig zurückbildeten.

Des Weiteren wollen wir in diesen Sitzungen mittels durch die Kopfhaut und den Schädel (transkraniell) gegebenen Magnetimpulsen mit Einzelreizen und Salvenreizen Erregungs- und Hemmungsvorgänge im Bereich der für die Arme zuständigen Gehirnzellen messen. Die transkranielle Magnetstimulation ist eine nicht-invasive und schmerzfreie Untersuchungsmethode.

Zu einem Stimulationstermin werden Sie eine wirksame, zu einem anderen eine nicht wirksame Stimulation erhalten, eine sogenannte Placebo-Stimulation. Um Erwartungseffekte zu vermeiden, wird Ihnen die Art der Stimulation nicht mitgeteilt. Selbstverständlich ist es Ihnen jederzeit möglich, ohne Angabe von Gründen und ohne dass Ihnen hieraus Nachteile entstehen, von der Teilnahme an der Studie zurückzutreten. Nachhaltige Wirkungen werden durch diese Untersuchung nicht angestrebt und sind auch nicht zu erwarten.

Eine Untersuchung umfasst voraussichtlich 4-5 Sitzungen, von jeweils 2-2,5 Stunde Gesamtdauer. Ein Abstand von mindestens einer Woche wird zwischen den Sitzungen eingehalten. Die Entlohnung beträgt 7 Euro pro begonnener Stunde. Wir streben an, dass jeder Proband/jede Probandin alle Untersuchungen durchläuft.

Selbstverständlich ist es Ihnen jederzeit möglich, ohne Angabe von Gründen und, ohne dass Ihnen hieraus Nachteile entstehen, von der Teilnahme an der Studie zurückzutreten. Nachhaltige Wirkungen werden durch diese Untersuchung nicht angestrebt und sind auch nicht zu erwarten. 


\section{Falls einer der folgenden Punkte auf Sie zutrifft, ist eine Teilnahme an der Studie nicht möglich:}

Herzschrittmacher

Metallimplantate im Kopfbereich (in den Kopfbereich eingesetztes Metall, z.B. Clips nach Operation eines intrazerebralen Aneurysmas (Gefäßaussackung im Bereich der Gehirngefäße), Implantation eine künstlichen Hörschnecke, eventuell auch Metallimplantate (Nägel, Platten, Schrauben) in anderen Körperabschnitten. Alter $<18$ oder $>55$ Jahre.

Hinweise auf eine chronische oder Residuen (Reste) einer neurologischen Erkrankung (Erkrankung des Nervensystems) in der Vorgeschichte.

Intrazerebrale Ischämien (Mangeldurchblutung des Gehirns/Schlaganfall/Blutungen) in der Vorgeschichte.

Hinweise auf epileptische Anfälle in der Vorgeschichte.

Hinweise auf Migräne

Vorliegen einer gravierenden internistischen (Erkrankung der inneren Organe) oder psychiatrischen (seelische Erkrankung) Vorerkrankung, insbesondere Schizophrenie (seelische Erkrankung mit Wahn, Trugwahrnehmungen und Störungen des Denkens) oder Manie (Erkrankung des Gemütes mit gehobener Stimmung). Schwangerschaft oder Stillperiode

$\begin{array}{cllrl}\text { Die aus } & \text { der Studie gewonnenen Daten werden anonymisiert } \\ \text { wissenschaftlich ausgewertet. } & \text { Alle Erfordernisse des Datenschutzes }\end{array}$
werden dabei beachtet. Zudem werden die Untersuchungstermine, Ihr Name und der Name der Studie in einer abteilungsinternen Datenbank dokumentiert. Sollten Sie als Folge der Studie Gesundheitsschädigungen erleiden, bitten wir Sie, uns dies unverzüglich mitzuteilen."

Prof. Dr. med. W. Paulus

Leiter der Abteilung

Klinische Neurophysiologie

Dr Michael Nitsche

Klinische Neurophysiologie

PD Dr Andrea Antal

Klinische Neurophysiologie 


\section{Literaturverzeichnis}

Anand S, Hotson J (2002): Transcranial magnetic stimulation: neurophysiological applications and safety. Brain Cogn 50, 366-386

Antal A, Boros K, Poreisz C, Chaieb L, Terney D, Paulus W (2008): Comparatively weak after-effects of transcranial alternating current stimulation (tACS) on cortical excitability in humans. Brain Stimulat 2008,1, 97-105

Antal A, Chaieb L, Moliadze V, Monte-Silva K, Poreisz C, Thirugnanasambandam N, Nitsche MA, Shoukier M, Ludwig H, Paulus W (2010): Brain-derived neurotrophic factor (BDNF) gene polymorphisms shape cortical plasticity in humans. (Brain Stimulat, im Druck)

Barker AT (1991): An introduction to the basic principles of magnetic nerve stimulation. $J$ Clin Neurophysiol 8, 26-37

Barker AT, Jalinous R, Freeston IL (1985a): Non-invasive magnetic stimulation of human motor cortex. Lancet 1985,1, 1106-1107

Barker AT, Freestone IL, Jalinous R, Merton PA, Morton HB (1985b): Magnetic stimulation of the human brain. J Physiol 369, 3P

Barker AT, Jalinous R, Freeston IL, Jarratt JA (1987): Magnetic stimulation of the human brain and peripheral nervous system: an introduction and the results of an initial clinical evaluation. Neurosurgery 20, 100-109

Bindman LJ, Lippold OC, Redfearn JW (1962): Long-lasting Changes in the Level of the Electrical Activity of the Cerebral Cortex produced by Polarizing Currents. Nature 196, 584-585 
Bindman LJ, Lippold OC, Redfearn JW (1964): The action of brief polarizing currents on the cerebral cortex of the rat (1) during current flow and (2) in the production of longlasting after-effects. J Physiol 172, 369-382

Bliss TV, Lomo T (1973): Long-lasting potentiation of synaptic transmission in the dentate area of the anesthetized rabbit following stimulation of the perforant path. $\mathrm{J}$ Physiol 232, 331-356

Boggio PS, Rigonatti SP, Ribeiro RB, Myczkowski ML, Nitsche MA, Pascual-Leone A, Fregni $F$ (2008): A randomized, double-blind clinical trial on the efficacy of cortical direct current stimulation for the treatment of major depression. Int $\mathrm{J}$ Neuropsychopharmacol $11,249-254$

Buonomano DV, Merzenich MM (1998): Cortical plasticity: from synapses to maps. Annu Rev Neurosci 21, 149-186

Brandt SA, Ploner CJ, Meyer BU (1997): Repetitive transkranielle Magnetstimulation. Nervenarzt 68, 778-784

Bromm B (1968): Sodium rectification in the subthreshold excitation as computed from the voltage clamp analysis. Pflugers Arch 302, 233-244

Buzsáki G, Draguhn A (2004): Neuronal oscillations in cortical networks. Science 304, 1926-1929

Carney MW (1969): Negative polarization of the brain in the treatment of manic states. Ir J Med Sci 2, 133-135

Castro-Almancos MA, Calcagnotto ME (1999): Presynaptic long-term potentiation in corticothalamic synapses. J Neurosci 19, 9090-9097 
Chaieb L, Kovacs G, Cziraki C, Greenlee M, Paulus W, Antal A (2009): Short-duration transcranial random noise stimulation induces blood oxygenation level dependent response attenuation in the human motor cortex. Exp Brain Res 198, 439-444

Chen R, Classen J, Gerloff C, Celnik P, Wassermann EM, Hallett M, Cohen LG (1997): Depression of motor cortex excitability by low-frequency transcranial magnetic stimulation. Neurology 48, 1398-1403.

Costain R, Readfearn JW, Lippold OC (1964): A controlled trail of therapeutic effects of polarization of the brain in depressive illness. Br J Psychiatr 110, 786-99

Cowansage KK, Ledoux JE, Monfils MH (2009): Brain-Derived Neurotrophic Factor: A Dynamic Gatekeeper of Neural Plasticity. (Curr Mol Pharmacol, im Druck)

Di Lazzaro V, Pilato F, Saturno E, Oliviero A, Dileone M, Mazzone P, Insola A, Tonali PA, Ranieri F, Huang YZ et al. (2005): Theta-burst repetitive transcranial magnetic stimulation suppresses specific excitatory circuits in the human motor cortex. J Physiol $565,945-950$

Edgley SA, Eyre JA, Lemon RN, Miller S (1997): Comparison of activation of corticospinal neurons and spinal motor neurons by magnetic and electrical transcranial stimulation in the lumbosacral cord of the anaesthezised monkey. Brain 120, 839-853

Elbert T, Pantev C, Wienbruch C, Rockstroh B, Taub E (1995): Increased cortical representation of the fingers of the left hand in string players. Science 270, 305-307

Eysel TU, Eyding D, Schweigart G (1998): Repetitive optical stimulation elicits fast resceptive field changes in mature visual cortex. Neuroreport 9, 949-954

Fanselow EE, Reid AP, Nicolelis MA (2000): Reduction of pentylenetetrazole-induced seizure activity in awake rats by seizure-triggered trigeminal nerve stimulation. $J$ Neurosci 20, 8160-8168 
Ferrier D (1875): Experiments on the brain of monkeys. Proc R Soc Lond 23, 409-430

Fitzgerald PB, Brown TL, Daskalakis ZJ, Chen R, Kulkarni J (2002): Intensity-dependent effects of $1 \mathrm{~Hz}$ rTMS on human corticospinal excitability. Clin Neurophysiol 113, 11361146

Foerster O (1936): The motor cortex in man in light of Hughlingss Jackson's doctrines. Brain 59, 135-159

Franca M, Koch G, Mochizuki H, Huang YZ, Rothwell JC (2006): Effects of theta burst stimulation protocols on phosphene threshold. Clin Neurophysiol 117, 1808-1813

Fregni F, Boggio PS, Mansur CG, Wagner T, Ferreira MJ, Lima MC, Rigonatti SP, Marcolin MA, Freedman SD, Nitsche MA et al. (2005): Transcranial direct current stimulation of the unaffected hemisphere in stroke patients. Neuroreport $\underline{16}, 1551-1555$

Fritsch G, Hitzig E (1870): Über die elektrische Erregbarkeit des Grosshirns. Archiv Anat Physiol Wiss Med 37, 300-332

Fuentes R, Petersson P, Siesser WB, Caron MG, Nicolelis MA (2009): Spinal cord stimulation restores locomotion in animal models of Parkinson's disease. Science 323, 1578-1582

Grossheinrich N, Rau A, Pogarell O, Hennig-Fast K, Reinl M, Karch S, Dieler A, Leicht G, Mulert C, Sterr A et al. (2009): Theta burst stimulation of the prefrontal cortex: safety and impact on cognition, mood, and resting electroencephalogram. Biol Psychiatry 65, 778-784

Hattori Y, Moriwaki A, Hori Y (1990): Biphasic effects of polarizing current on adenosinesensitive generation of cyclic AMP in rat cerebral cortex. Neurosci Lett 116, 320-324 
Hess G, Donoghue JP (1994): Long-term potentiation of horizontal connections provides a mechanism to reorganize cortical motor maps. J Neurophysiol 71, 2543-2547

Huang YZ, Edwards MJ, Rounis E, Bhatia KP, Rothwell JC (2005): Theta burst stimulation of the human motor cortex. Neuron 45, 201-206

Huang YZ, Chen RS, Rothwell JC, Wen HY (2007): The after-effect of human theta burst stimulation is NMDA receptor dependent. Clin Neurophysiol 118, 1028-1032

Huang YZ, Sommer M, Thickbroom G, Hamada M, Pascual-Leone A, Paulus W, Classen J, Peterchev AV, Zangen A, Ugawa Y (2009): Consensus: New methodologies for brain stimulation. Brain Stimulat 2009,2, 2-13

Hummel F, Cohen LG (2005): Improvement of motor function with noninvasive cortical stimulation in a patient with chronic stroke. Neurorehabil Neural Repair 19, 14-19

Islam N, Aftabuddin M, Moriwaki A, Hattori Y, Hori Y (1995): Increase in the calcium level following anodal polarization in the rat brain. Brain Res 684, 206-208

Kanai R, Chaieb L, Antal A, Walsh V, Paulus W (2008): Frequency-dependent electrical stimulation of the visual cortex. Curr Biol 18, 1839-1843

Krug M, Lossner B, Ott T (1984): Anisomycin blocks the late phase of long-term potentiation in the dentate gyrus of freely moving rats. Brain Res Bull 13, 39-42

Larson J, Lynch G (1986): Induction of synaptic potentiation in hippocampus by patterned stimulation involves two events. Science 232, 985-988

Leyton ASF, Sherrington CS (1917): Observations on the exitable cortex: Oft the chimpanzee, orang-utan, and gorilla. Q J Exp Physiol 11, 135-222 
Liebetanz D, Nitsche MA, Tergau F, Paulus W (2002): Pharmacological approach to the mechanisms of transcranial DC-stimulation-induced after-effects of human motor cortex excitability. Brain 125, 2238-2247

Lisman JE, Idiart MA (1995): Storage of $7+/-2$ short-term memories in oscillatory subcycles. Science $267,1512-1515$

Lolas F (1977): Brain polarization: behavioral and therapeutic effects. Biol Psychiatry $114,635-637$

McCreery DB, Agnew WF, Yuen TG, Bullara L. (1990): Charge density and charge per phase as cofactors in neural injury induced by electrical stimulation. IEEE Trans Biomed Eng 37, 996-1001

Merton PA, Morton HB (1980): Stimulation of the cerebral cortex in the intact human subject. Nature 285, 227

Mochizuki H, Franca M, Huang YZ, Rothwell JC (2005): The role of dorsal premotor area in reaction task: comparing the "virtual lesion" effect of paired pulse or theta burst transcranial magnetic stimulation. Exp Brain Res 167, 414-421

Mori F, Codecà C, Kusayanagi H, Monteleone F, Boffa L, Rimano A, Bernardi G, Koch G, Centonze D (2009): Effects of intermittent theta burst stimulation on spasticity in patients with multiple sclerosis. (Eur J Neurol, im Druck)

Mori F, Codecà C, Kusayanagi H, Monteleone F, Buttari F, Fiore S, Bernardi G, Koch G, Centonze D (2010): Effects of Anodal Transcranial Direct Current Stimulation on Chronic Neuropathic Pain in Patients With Multiple Sclerosis. (J Pain, im Druck)

Nitsche MA, Paulus W (2000): Excitability changes induced in the human motor cortex by weak transcranial direct current stimulation. J Physiol 527, 633-639 
Nitsche MA, Liebetanz D, Lang N, Antal A, Tergau F, Paulus W (2003): Safety criteria for transcranial direct current stimulation (tDCS) in humans. Clin Neurophysiol 114, 2220-2222

$\underline{\text { Nitsche MA, Doemkes S }}$, Karaköse T, Antal A, Liebetanz D, Lang N, Tergau F, Paulus W (2007): Shaping the effects of transcranial direct current stimulation of the human motor cortex. J Neurophysiol 97, 3109-3117

Nitsche MA, Cohen LG, Wassermann EM, Priori A, Lang N, Antal A, Paulus W, Hummel F, Boggio PS, Fregni F et al. (2008): Transcranial direct current Stimulation: State of the Art 2008. Brain Stimulat 2008,1, 206-223

Nowak DA, Hoffmann U, Connemann BJ, Schönfeldt-Lecuona C (2006): Epileptic seizure following $1 \mathrm{~Hz}$ repetitive transcranial magnetic stimulation. Clin Neurophysiol $117,1631-1633$

Oldfield RC (1971): The assessment and analysis of handedness: the Edinburgh inventory. Neuropsychologia 9, 97-113

Pascual-Leone A, Houser CM, Reese K, Shotland LI, Grafman J, Sato S, Valls-Solé J, Brasil-Neto JP, Wassermann EM, Cohen LG et al. (1993): Safety of rapid-rate transcranial magnetic stimulation in normal volunteers. Electroencephalogr Clin Neurophysiol 89, 120-130

Pascual-Leone A, Valls-Sol J, Wassermann EM, Hallett M (1994): Responses to rapidrate transcranial magnetic stimulation of the human motor cortex. Brain 117, 847-858

Pascual-Leone A, Tormos JM, Keenan J, Tarazona F, Cañete C, Catalá MD. (1998): Study and modulation of human cortical excitability with transcranial magnetic stimulation. J Clin Neurophysiol 15, 333-343 
Peinemann A, Reimer B, Löer C, Quartarone A, Münchau A, Conrad B, Siebner HR (2004): Long-lasting increase in corticospinal excitability after 1800 pulses of subthreshold $5 \mathrm{~Hz}$ repetitive TMS to the primary motor cortex. Clin Neurophysiol 115, 1519-1526

Penfield W, Boldrey E (1937): Somatic motor and sensory representation in the cerebral cortex of man as studied by electrical stimulation. Brain $60,389-443$

Penfield W, Rasmussen T: The cerebral cortex of man. The MacMillan Company, New York 1950

Poreisz C, Boros K, Antal A, Paulus W (2007): Safety aspects of transcranial direct current stimulation concerning healthy subjects and patients. Brain Res Bull 72, 208-214

Poreisz C, Paulus W, Moser T, Lang N (2009): Does a single session of theta-burst transcranial magnetic stimulation of inferior temporal cortex affect tinnitus perception? BMC Neurosci 2009 10, 54

Purpura DP, McMurtry JG (1965): Intracellular activities and evoked potential changes during polarization of motor cortex. J Neurophysiol 28, 166-185

Quartarone A, Bagnato S, Rizzo V, Morgante F, Sant'angelo A, Battaglia F, Messina C, Siebner HR, Girlanda P (2005): Distinct changes in cortical and spinal excitability following high-frequency repetitive TMS to the human motor cortex. Exp Brain Res 161, 114-124

Roelfsema PR, Engel AK, König P, Singer W (1997): Visomotor integration is associated with zero time-lag synchronization among cortical areas. Nature 385, 157-161

Rossini PM, Pauri F (2000): Neuromagnetic integrated methods tracking human brain mechanisms of sensorimotor areas 'plastic' reorganisation. Brain Res Brain Res Rev 33, $131-154$ 
Rossini PM, Desiato MT, Lavaroni F, Caramia MD (1991): Brain excitability and electroencephalographic activation: non-invasive evaluation in healthy humans via transcranial magnetic stimulation. Brain Res 567, 111-119

Singer W (1999): Neuronal synchrony: a versatile code for the definition of relations? Neuron 24, 111-125

Schubert M (1997): Klinische und experimentelle Aspekte der Magnetstimulation: Z EEG EMG 28, 114-118

Siebner HR, Rothwell J (2003): Transcranial magnetic stimulation: new insights into representational cortical plasticity. Exp Brain Res 148, 1-16

Steinhoff BJ, Tumani H, Otto M, Mursch K, Wiltfang J, Herrendorf G, Bittermann HJ, Felgenhauer K, Paulus W, Markakis E (1999): Cisternal S100 protein and neuronspecific enolase are elevated and site-specific markers in intractable temporal lobe epilepsy. Epilepsy Res 36, 75-82

Talelli P, Greenwood RJ, Rothwell JC (2007): Exploring Theta Burst Stimulation as an intervention to improve motor recovery in chronic stroke. Clin Neurophysiol 118, 333-342

Terney D, Chaieb L, Moliadze V, Antal A, Paulus W (2008): Increasing human brain excitability by transcranial high-frequency random noise stimulation. J Neurosci 28 , 14147-14155

Touge T, Gerschlager W, Brown P, Rothwell JC (2001): Are the after-effects of lowfrequency rTMS on motor cortex excitability due to changes in the efficacy of cortical synapses? Clin Neurophysiol 112, 2138-2145

Toyoda H, Zhao MG, Zhuo M (2006): NMDA receptor-dependent long-term depression in the anterior cingulate cortex. Rev Neurosci 17, 403-413 
Wassermann EM (1998): Risk and safety of repetitive transcranial magnetic stimulation: report and suggested guidelines from the International Workshop on the Safety of Repetitive Transcranial Magnetic Stimulation, June 5-7, 1996. Electroencephalogr Clin Neurophysiol 108, 1-16

Wassermann EM, Cohen LG, Flitman SS, Chen R, Hallett M. (1996a): Seizures in healthy people with repeated "safe" trains of transcranial magnetic stimuli. Lancet 347 , 825-826

Wassermann EM, Grafman J, Berry C, Hollnagel C, Wild K, Clark K, Hallett M (1996b): Use and safety of a new repetitive transcranial magnetic stimulator. Electroencephalogr Clin Neurophysiol 101, 412-417

Yuen TG, Agnew WF, Bullara LA, Jacques S, McCreery DB (1981): Histological evaluation of neural damage from electrical stimulation: considerations for the selection of parameters for clinical application. Neurosurgery 9, 292-293

Yuste R, Bonhoeffer T (2001): Morphological changes in dendritic spines associated with long-term synaptic plasticity. Annu Rev Neurosci 24, 1071-1089 


\section{Danksagung}

Ich danke der Abteilung der Klinischen Neurophysiologie der Universitätsmedizin Göttingen unter der Leitung von Prof. Dr. med. W. Paulus sowie der Gruppenleitung von Frau Prof. Dr. rer. nat. Andrea Antal für die Bereitstellung der technischen Gerätschaften und der wissenschaftlichen und finanziellen Mittel. Frau Dr. rer. nat. Vera Moliadze gilt mein besonderer Dank für die Betreuung, die wertvollen Anregungen und die geduldige Unterstützung bei technischen und fachlichen Fragen. Insbesondere danken möchte ich Frau Dipl. psych. Anna-Sophia Fritzsche für die perfekte Hilfe bei der statistischen Auswertung und Motivation, ohne welche die Erstellung der Arbeit nicht möglich gewesen wäre. Weiterhin gilt mein Dank den freiwilligen Probanden und Probandinnen, die durch Ihre Beteiligung diese Arbeit erst ermöglicht haben. 